\title{
II. Die Entnazifizierung in Württemberg-Hohenzollern nach den Richtlinien der französischen Militärregierung
}

\section{Die Einsetzung des Staatssekretariats für die französisch besetzte Zone Württembergs und Hohenzollerns in Tübingen}

Da es die Franzosen im Gegensatz zum Military Government der US-Zone einerseits aus prinzipiellen Erwägungen heraus nicht für angebracht hielten, die politische Säuberung des deutschen öffentlichen Lebens in eigener Regie durchzuführen und andererseits die Überwindung der von Ort zu Ort differierenden Säuberungsansätze durch die Anlegung einheitlicher Maßstäbe für das französische Kontrollgebiet schon allein aus Gründen der Gleichbehandlung unabdingbar war, kam für die Militärregierung als Partner einer systematischen Entnazifizierung nur eine deutsche Verwaltung in Frage, deren Kompetenzen sich auf das ganze französisch besetzte Gebiet Württembergs erstreckte.

Da Frankreich in den Verhandlungen um die Zuweisung eines Besatzungsgebietes im Südwesten Deutschlands seine Vorstellung nicht gegen die militärisch-logistischen Interessen der USA durchsetzen konnte, hatte sich die Regierung in Paris mit der Ưbernahme Südwürttembergs als Kompensationsobjekt für die favorisierte, aber nicht erreichbare L.ösung einer Einbeziehung von ganz Baden in die französische Besatzungszone zufrieden geben müssen. Dies war zweifellos eine Enttäuschung. De Gaulle hatte ja vor allem das Land Baden in sein politisches Konzept einbezogen, dem für die linksrheinischen Gebiete kaum verschleierte annexionistische Vorstellungen zugrunde lagen ${ }^{1}$ und das unter Wiederbelebung historischer Reminiszenzen die Etablierung einer ausgedehnten französischen Einflußsphäre entlang des rechten Rheinufers in Baden anstrebte. Um sich die Mitwirkung prominenter badischer Repräsentanten bei solchen Plänen zu sichern, haben sich Vertreter der französischen Militärregierung in charakteristischer Hintanstellung der politischen Säuberung gegenüber territorialen und wirtschaftlichen Interessen offenbar nicht gescheut, das Angebot einer „,konzilianten“ Entnazifizierung in Baden zu machen ${ }^{2}$. Wie immer de Gaulle sich die besondere Orientierung Badens nach Frankreich vorgestellt haben mag, die Grenzziehung im Südteil der französischen Zone kam diesen Vorstellungen nicht entgegen. Von beiden Ländern waren nur deren schwach industrialisierte südlichen Teile ohne ihre bedeutenden Zentren im Norden, Karlsruhe und Stuttgart, in den französischen Einflußbereich einbezogen. Die Hoffnung auf einen Wandel in der Struktur der alliierten Deutschland-Kontrolle, insbesondere auf einen Rückzug der Amerikaner aus Europa, den damals noch viele für möglich hielten, mag es den Franzosen erleichtert

${ }^{1}$ Frankreich erstrebe dort, wie es de Gaulle in seiner Baden-Badener Rede v. 5.10.1945 ausdrückte, „une présence, un contrôle indefini“. La Revue de la Zone Française, Nr. 1, 15.11. 1945, S. 9 (IfZ-Archiv).

2 Dorn, Inspektionsreisen, S. 62. Von ähnlichen Praktiken in der Pfalz berichtet Wünschel, Separatismus, S. $123 \mathrm{f}$. 
haben, die Arbeit erst einmal in den Gebieten zu beginnen, die ihnen verblieben waren ${ }^{3}$. Auch so waren die Probleme der Installation einer funktionierenden Zonenadministration noch verwickelt genug. Das ließ sogar de Gaulle in einer Rede vor Besatzungsoffizieren anklingen. Ihm sei zu Ohren gekommen, sagte er, „que le Gouvernement Militaire avait eu des difficultés pour s'installer"“4.

Nach dem Scheitern des französischen Baden-Konzeptes begann mit der Errichtung der Délégation Supérieure für das französisch besetzte Gebiet Württembergs und Hohenzollerns die Konsolidierung des Erreichbaren. Nun mußte sich auch jener Transformierungsprozeß beschleunigen, durch den die Tübinger Landesdelegation nach und nach in eine eigenständige, ausschließlich der französischen Besatzungsmacht verantwortliche Auftragsverwaltung umgewandelt wurde. Begleitet wurde diese Entwicklung von der stets wiederkehrenden Versicherung der französischen Verantwortlichen, die Einheit Württembergs solle durch die Einrichtung der Tübinger Separatverwaltung nicht angetastet werden ${ }^{5}$. Hinter diesen Versicherungen stand einerseits sicherlich die französische Hoffnung, schließlich doch noch ganz Württemberg übernehmen zu können ${ }^{6}$, andererseits waren diese Erklärungen aber auch geeignet, den beteiligten deutschen Politikern ihre Mitwirkung an der sich abzeichnenden Teilung des Landes zu erleichtern. Diesen Zusagen kam in der Folgezeit zwar keinerlei praktische Bedeutung zu, doch trugen sie dazu bei, daß die Konsequenzen der Aufteilung Württembergs auf zwei Besatzungszonen von Behörden und Bevölkerung erst realitiv spät erkannt wurden ${ }^{7}$. Während dieser Entwicklung herrschte innerhalb der Militärregierung offenbar selbst eine gewisse Konzeptionslosigkeit, denn bei der Installierung des Tübinger Staatssekretariats fand man erst spät zu einer eindeutigen Haltung. In den Verhandlungen zwischen Carlo Schmid und dem Kabinettschef des Gouverneurs, Oberst Corbin de Mangoux, war man nämlich Anfang Oktober zunächst ausdrücklich übereingekommen ${ }^{8}$, daß dem französischen Gouverneur in Tübingen am 13. des Monats ${ }^{9}$ ein Beglaubigungsschreiben von Ministerpräsident Rein-

${ }^{3}$ Carlo Schmid überliefert, daß man sich innerhalb der französischen Militärregierung mit solchen Gedanken getragen hatte; Konstanzer, Entstehung, S. 84.

${ }^{4}$ La Revue de la Zone Française, Nr. 1, 15. 11. 1945, S. 10 (IfZ-Archiv).

5 Gouverneur Widmer zu Reinhold Maier auf einer Besprechung in Tübingen am 13.10. 1945; vergl. dazu den Bericht von Staatsrat Wittwer an die amerikanische Militärregierung in Stuttgart v. 15. 10. 1945 (HStASt EA 1/20 A8/2), das Schreiben C. Schmids an das StaMi Stuttgart v. 22.10.1945 (HStASt EA 1/20 A8/7), sowie C. Schmids „Niederschrift zur Vorgeschichte der Errichtung des Staatssekretariats" v. 17.10.1945 (SIG, Wü 2, II, 600/1/6). S.a. C. Schmids Niederschrift „über die Eröffnungssitzung des Staatssekretariats“v. 17. 10. 1945 (SIG, Wü 2, II, 600/1/7). $\quad{ }^{6}$ Information Gebhard Müller. S.a. Konstanzer, Entstehung, S. 84.

7 Vergl. Eschenburg, Anfänge, S. 265.

8 C.Schmids „Niederschrift zur Vorgeschichte der Errichtung des Staatssekretariats“ v. 17. 10. 1945; SIG, Wü 2, II, 600/1/6. Dieser Lösung hatte laut Niederschrift auch Oberst Dawson, der Chef der amerikanischen Milreg von Württemberg-Baden, zugestimmt.

9 Dieses Datum gibt C. Schmid in seinem Schreiben an das StaMi in Stuttgart v. 22. 10. 1945, betr. die „Errichtung des Staatssekretariats für das franz. besetzte Gebiet Württembergs und Hohenzollerns in Tübingen“ an (HStASt EA 1/20 A8/7). In der in Anm. 5 zitierten „Niederschrift zur Vorgeschichte..." nennt er den 12. Oktober als Übergabetermin. 
hold Maier ${ }^{10}$ überreicht werden sollte, mit dem Staatsrat Schmid als General-Verantwortlicher für die einzelnen Landesdelegationen zum „Vertreter des Staatsministeriums bei der franz. Militärregierung “ bestellt werden sollte ${ }^{11}$. Doch dann erklärte de Mangoux in der Vorbereitungsbesprechung, einen Tag vor der vereinbarten Ubergabe des Dokuments, dem überraschten Schmid plötzlich, die französische Militärregierung könne es bei dem vorgesehenen Modus nicht mit ihrer Souveränität und Autonomie vereinbaren, daß die südwürttembergische Verwaltung aufgrund eines „Verwaltungsaktes organisiert werde, für den der Herr Ministerpräsident erst die amerikanische Genehmigung einholen müsse." Das Beglaubigungsschreiben wurde nicht entgegengenommen ${ }^{12}$. Die Anwesenheit Reinhold Maiers bei der Eröffnungssitzung des Staatssekretariats hatte keine staatsrechtliche Relevanz mehr. Vor dem Stuttgarter Kabinett kennzeichnete Maier das Tauziehen um den Status der Tübinger „Verbindungsbehörde“, wie er sie noch immer nannte, mit den Worten: „Ursprünglich wollten wir die Vertreter bestellen, dann wollten sie die Franzosen ernennen und ich sollte sie einsetzen, schließlich war es nur eine Beiwohnung zur Amtseinsetzung, die eigentlich niemand vornahm, immerhin ein symbolischer Akt, um die Einheit Württembergs zu dokumentieren “13. Auch Carlo Schmid, der neugewählte Vorsitzende des Staatssekretariats, ließ es sich angelegen sein, wo immer sich Gelegenheit dazu bot, wenigstens den moralischen Anspruch der neuen Verwaltung auf eine Revision der Teilung Württembergs zu unterstreichen, die unter den gegebenen Umständen nicht zu verhindern gewesen war $^{14}$.

Im Staatssekretariat, auf dessen organisatorische und personelle Einzelheiten hier nicht näher eingegangen zu werden braucht, nahm Carlo Schmid als Vorsitzender und Landesdirektor zweier Ressorts (Justiz, Kultus) sowie aufgrund seines großen persön-

${ }^{10}$ Schreiben Reinhold Maiers an Gouverneur Widmer v. 9. 10. 1945; SIG, Wü 2, II, 600/1/2.

11 Schreiben C. Schmids an das StaMi in Stuttgart v. 22. 10. 1945; ebd.

12 Ebd. Konstanzer meint, es ,sei nicht ganz verständlich“ (Entstehung, S. 27), wie die Landesregierung in Stuttgart mit der Akzeptierung des Beglaubigungsschreibens durch die französische Militärregierung habe rechnen können. Eben diesen Modus, „daß am 13.10.1945 das vom Herrn Ministerpräsidenten für ihn [Schmid] ausgefertigte Beglaubigungsschreiben dem Gouverneur in Tübingen überreicht werden sollte" (Schmid in seinem Schreiben an das StaMi v. 22. 10. 1945), hatten Schmid und de Mangoux aber Anfang Oktober ausdrücklich vereinbart. Der Grund für den französischen Sinneswandel wird in den verstärkten amerikanischen Aktivitäten zur Fusion Nordwürttembergs-Nordbaden und zur Bildung des Länderrats der amerikanischen Zone zu suchen sein.

13 5. Sitzung des StaMi in Stuttgart am 17. 10. 1945; HStASt EA 1/20, C1/32 - 1945. Die Rede Reinhold Maiers auf der Tübinger Eröffnungssitzung des Staatssekretariats am 16. 10. 1945, in: HStASt EA 1/20 A8/3. Ein eigentlich konstituierender Akt durch die französische Milreg war unterblieben; Gouverneur Widmer nahm die Bildung des Staatssekretariats lediglich ,zur Kenntnis“. S. „Niederschrift über die Eröffnungssitzung ..." von C. Schmid v. 17. 10. 1945; SIG, Wü 2, II, 600/1/6. S.a. Maier, Ende und Wende, S. 362.

14 Vergl. das Statut des Staatssekretariats v. 30.10.1945 im Amtsblatt für das französisch besetzte Gebiet Württembergs und Hohenzollerns, S. 1. Ferner die Rede Carlo Schmids auf der 1. Lrtg in Tübingen am 3. 11. 1945. Ausführlich zum Selbstverständnis des Staatssekretariats, Eschenburg, Anfänge, S. 266 und Konstanzer, Entstehung S. 31 ff. 
lichen Ansehens, das er bei den französischen Militärbehörden geno $\beta^{15}$, eine überragende Stellung ein. Von der Militärregierung in Stuttgart war er allerdings bei der Bildung der dortigen Landesregierung nicht nur nicht berücksichtigt, sondern sogar aus seinem Amt als Landesdirektor der Kultusverwaltung entlassen und einige Tage inhaftiert worden ${ }^{16}$. Diese Vorkommnisse haben einigen Kreisen in Frankreich Anlaß zu Spekulationen über seine Tätigkeit als Kriegsverwaltungsrat in Lille während der deutschen Besetzung Frankreichs gegeben ${ }^{17}$. Der französische Geheimdienst ging diesen Vorwürfen nach und rekonstruierte Schmids Tätigkeit in Frankreich minutiös. Es blieb nach dieser Untersuchung innerhalb des Gouvernement Militaire auch nicht der Schatten eines Verdachtes gegen den Vorsitzenden des Staatssekretariats in Tübingen $^{18}$.

Mit der Etablierung des Staatssekretariats in Tübingen hatte der monatelang sich hinziehende, stetig sich vertiefende Prozeß der verwaltungsmäßigen Teilung Württembergs seinen Abschluß gefunden. Sowohl im amerikanisch besetzten Nordwürttemberg als auch im französisch besetzten Süden des Landes bestanden nun zwei eigenständige, voneinander unabhängige deutsche Auftragsverwaltungen. Erst jetzt, ein halbes Jahr nach der Besetzung, verfügte die französische Besatzungsmacht in Südwürttemberg über ein Instrument, mit dem sie die vorangegangene, von unterschiedlichen ad-hoc-Maßnahmen geprägte und in die verschiedensten Sonderentwicklungen zerfaserte Periode der allgemeinen Konzeptionslosigkeit beenden und mit einer einheitlichen, allgemein verbindlichen Umsetzung ihrer Vorstellungen in allen Bereichen der Besatzungspolitik beginnen konnte. Die einheitliche Durchführung der politischen Säuberung, für die Gouverneur Widmer gleich in der Eröffnungssitzung des Staatssekretariats neue Richtlinien ankündigte, war ein wesentliches Element dieses neuen Anlaufes, für den nun in Südwürttemberg die Voraussetzungen geschaffen waren.

2. Die französischen Richtlinien zur politischen Säuberung des öffentlichen

Dienstes

Als die französischen Militärbehörden am 19. Oktober 1945 die Richtlinien für die politische Säuberung in Südwürttemberg und Hohenzollern herausgaben, hatte sich die Haltung Frankreichs im Alliierten Kontrollrat sowie das allgemeine Klima der Entnazifizierung in einer Weise entwickelt, die es der französischen Militärregierung nicht erlaubte, den Säuberungsmodus aus der Zeit des Stuttgarter Interims wieder aufzunehmen. Aufgrund des starken Gefälles in der Intensität der politischen Säuberung zwischen französischem und amerikanischem Besatzungsgebiet hatte sich die

\footnotetext{
15 Vergl. Eschenburg, Anfänge, S. 270.

16 Ebd., S. 265. S.a. Maier, Ende und Wende, S. 332.

17 Guy Mollet (S.F.I.O.) forderte von der SPD Aufklärung über die Rolle Schmids während der deutschen Besetzung Frankreichs; Korff, Le revirement, S. 190. Vergl. a. Morin, Allemagne, S. 75 und Pudack, Deutschlandpolitik, S. 194.

18 Information Eydoux.
} 
französische Zone inzwischen den Ruf erworben, ein „Eldorado der Duldsamkeit“ für politisch Belastete zu sein ${ }^{19}$. Ein Blick auf die unterschiedliche Säuberungspraxis in beiden Zonen zeigt, daß diese Einschätzung für das Jahr der Besetzung nicht unbegründet war, weil die Entnazifizierungsmaßnahmen in der amerikanischen Zone unter dem anhaltenden Druck der öffentlichen Meinung in den USA ja Zug um Zug solange verschärft worden waren, bis sie gegen Jahresende vollends ,planlos“20 ausuferten. Bei einem allzu weiten Zurückbleiben hinter dem amerikanischen Säuberungsstandard waren wegen der Unruhe, die diese Art von französischer Großzügigkeit in Nordwürttemberg auslösen mußte ${ }^{21}$, ernste Spannungen zwischen den beiden Besatzungsmächten in Württemberg nicht mehr auszuschließen. General Eisenhower hatte sich bereits über „die Laxheit der Franzosen“ beschwert ${ }^{22}$. Vor allem aber bestand die Gefahr, daß sich die französische Position im Alliierten Kontrollrat weiter verschlechterte. Denn dort hatte Frankreich die Einrichtung deutscher Zentralverwaltungen, wie sie das Potsdamer Abkommen vorsah, durch sein Veto blockiert und dabei eine Provokation der übrigen Alliierten in Kauf genommen. Etwa zur gleichen Zeit unternahmen die Amerikaner einen Vorstoß zur Vereinheitlichung der politischen Säuberung in Deutschland. Sie schlugen dem Kontrollrat die U. S.-Direktive vom 7. Juli 1945 zur Beschlußfassung vor ${ }^{23}$. Für die Franzosen konnte es in dieser Phase nicht ratsam sein, in dem höchsten alliierten Kontrollgremium noch mehr Konfliktstoff anzuhäufen ${ }^{24}$. Genau darauf wäre es aber hinausgelaufen, wenn die französischen Zonenbehörden ihre alliierten Partner diesmal mit dem Erlaß von Säuberungsbestimmungen brüskiert hätten, die mit den Vorstellungen, die gerade in Form einer Kontrollratsdirektive zur gemeinsamen alliierten Entnazifizierungspolitik erhoben werden sollten, nicht einmal mehr entfernt etwas gemein hatten. Man hätte auf französischer Seite riskiert, den Kredit für zukünftige interalliierte Verhandlungen im Kontrollrat vollends zu überziehen und dadurch die eigenen Interessen in anderen Bereichen der Besatzungspolitik zu gefährden. Da man diesen übergeordneten Interessen Rechnung zu tragen hatte, zu einer substantiellen Annäherung an die amerikanische Säuberungspolitik aber keine Bereitschaft bestand, blieb der französischen Militärregierung nur ein Ausweg aus diesem Dilemma: der groß angelegte Bluff.

$\overline{19}$ 9. Lrtg in Saulgau am 6.7.1946, S. 47. Vergl. die Außerung Schmids in der 3. Sitzung des Stuttgarter StaMi am 5. 10.1945, ,daß nach Tübingen auch hier [politisch] beanstandete Beamte geschickt werden könnten." HStASt, C 1/32, 1945. Ferner Dorn, Inspektionsreisen, S. 64.

20 Niethammer, Entnazifizierung, S. 247.

21 Staatskommissar Künzel berichtete auf der 9. Lrtg in Saulgau am 6. 7.1946 von der öffentlichen Klage Reinhold Maiers, daß die Durchführung der politischen Säuberung ,,immer mehr psychologisch unerträglich“ (Künzel) werde, solange in Südwürttemberg viel großzügigere Maßstäbe angelegt würden.

22 C. Schmid auf der 6. Sitzung des Stuttgarter StAMi am 24. 10. 1945. HStASt C 1/32, 1945.

23 Friedrich, American Experiences, S. 259 ff.; Griffith, Denazification Program, S. 129. Mit einigen „escape clauses" versehen (Griffith), die schließlich doch in jeder Zone ein eigenes Vorgehen ermöglichten, wurde sie als Kontrollrats-Direktive Nr. 24 am 12.1. 1946 verabschiedet (J. O., S. 228).

${ }^{24}$ Diesen Eindruck hatte man, nach einer Information Gebhard Müllers an den Verfasser, auch im Tübinger Staatssekretariat gewonnen. 
Die Entnazifizierungs-Richtlinien, die dem Staatssekretariat am 19. Oktober 1945 zugingen, bezogen sich nur auf die politische Säuberung der öffentlichen Verwaltung ${ }^{25}$. Darin schrieb die Militärregierung einen zweistufigen Säuberungsapparat vor: Die untere Ebene in diesem System bildeten die sogenannten Kreisuntersuchungsausschüsse (KRUAS), die in jedem Landkreis eingerichtet werden mußten. Sie sollten sich aus fünf ständigen Mitgliedern von „Vertretern politischer, konfessioneller und gewerkschaftlicher Kreise“ zusammensetzen. Hinzugezogen werden mußten jeweils ein bis drei Vertreter des Verwaltungszweiges, dessen Beamte und Angestellte gerade zur Überprüfung anstanden. Während diese durch die zuständigen Landesdirektionen ernannt wurden, waren die fünf ständigen Vertreter im KRUA durch die Landesdirektion des Innern zu benennen. Aus einer internen Dienstanweisung des Délégué Supérieur an seine Kreisdelegierten ${ }^{26}$ geht hervor, daß man auf französischer Seite peinlich darauf bedacht war, den KRUAS den Anschein weitgehender Eigenständigkeit zu geben. Dort hieß es nämlich, der Délégué de Cercle solle mit aller nötigen Sorgfalt die Arbeit der Ausschüsse überwachen, ,jedoch haben Sie in einer Weise vorzugehen, daß diese Ausschüsse in den Augen der Öffentlichkeit den Anschein allergrößter Unabhängigkeit gegenüber den französischen Behörden behalten“ ${ }^{\text {27. }}$.

Der KRUA, der die Untersuchungen in eigener Verantwortung einzuleiten hatte, sollte die Angaben der Beamten in ihren Fragebögen überprüfen; darüber hinaus konnte er eigene Ermittlungen zur politischen Vergangenheit des Kandidaten anstellen. Anhand der gewonnenen Erkenntnisse mußte er dann die Fragebogen nach zwei Gesichtspunkten klassifizieren, und zwar zunächst in diejenigen Fälle, bei denen bestimmte Formalbelastungen gemäß einer beiliegenden Liste zutrafen. Die Militärregierung forderte die autoinatische Entlassung dieser Gruppe. Die andere Kategorie umfaßte alle die Beamten, auf die zwar keine der aufgeführten formalen Belastungsmerkmale zutraf, die wegen ihrer Haltung zum Nationalsozialismus aber dennoch mit Sanktionen belegt werden sollten. Diese abgestuften Sanktionen für die Beamten dieser Gruppe sollten die sogenannten Säuberungskommissionen, die zweite Stufe des Säuberungsapparates, verfügen. Bei jeder Landesdirektion in Tübingen mußte eine solche Kommission errichtet werden. Diese Gremien sollten sich ebenfalls aus Vertretern politischer, konfessioneller und gewerkschaftlicher Kreise zusammensetzen; sie mußten aber, wie zusätzlich bestimmt war, ,in ihrer Person, in ihrer Laufbahn oder in ihrem Vermögen durch das nationalsozialistische Regime gelitten haben." Die Kommissionen sollten sich bei ihren Entscheidungen auf das von den KRUAS vorbereitete Material stützen. Nach diesen Richtlinien war ein deutscher Entscheidungsspielraum also nur bei den Fällen gegeben, die nicht unter die Formalbelastungsmerkmale fielen. Mit Sanktionen belegt werden sollten alle, ,die Mitglieder der Partei oder Sympathisanten

${ }^{25}$ Schreiben des Délégué Supérieur an den Vorsitzenden des Staatssekretariats v. 19. 10. 1945; SIG, Wü 2, I, 610 und in Wü 15/269.

${ }^{26}$ Schreiben v. 25. 10.1945. Das militärregierungsinterne Schriftstück wurde in den Akten des Reutlinger KRUA aufgefunden; SIG, Wü 15/269.

$27, \ldots$ gardent aux yeux du public une apparence d'autonomie aussi grande que possible vis à vis des autorités françaises." Ebd. 
waren und ihre Haupttätigkeit oder den größten Teil derselben der nationalsozialistischen Propaganda oder Tätigkeit, gleich welcher Art gewidmet haben“, sowie jene Beamten und Angestellten, „die der Partei angehörten oder Sympathisanten waren und, ohne persönlich aktiv gewesen zu sein, der nationalsozialistischen Propaganda oder der Partei ihren Namen oder ihre finanzielle Unterstützung zur Verfügung gestellt haben oder die sich der Denunziation bei den Nazi-Behörden schuldig gemacht haben." Schien der Säuberungskommission eine der beiden nicht näher erläuterten Gummiformeln anwendbar, so konnte sie den betroffenen Beamten mit vier abgestuften Sanktionen belegen ${ }^{28}$. Die ausgesprochenen Sanktionen mußten, ebenso wie die personelle Besetzung der KRUAS und Kommissionen, vom Gouverneur genehmigt werden. Eine Berufungs- oder Revisionsmöglichkeit für den Betroffenen bestand nicht. Entlassene durften in keiner Verwaltung mehr angestellt werden. Besonders strenge Maßstäbe sollten bei den politischen Beamten angelegt werden; Spezialisten konnten trotz politischer Belastung im Amt belassen werden, wenn sie einem Beamten unterstellt wurden, der wegen seiner, ,antinationalsozialistische[n] Einstellung “ bekannt war. Allein diese Bestimmung eröffnete der Auftragsverwaltung schon ein praktikables Vorgehen, das die praktisch unterschiedslose Ausschaltung von Beamten nach Kriterien, wie sie in der amerikanischen Zone zu dieser Zeit noch in Geltung standen, vermied. In den Richtlinien hieß es ferner, es sei „nicht die Aufgabe der Kommissionen und der Ausschüsse, grundsätzlich alle ehemals mit dem Nationalsozialismus Sympathisierenden auszuschließen", sondern sie dadurch unschädlich zu machen, daß sie in ein „Abhängigkeitsverhältnis“ von Beamten gebracht würden, die durch ihre ,antinazistische Überzeugung“ hervorgetreten seien. Abschließend ordnete die Militärregierung an, daß die Säuberung am 1. November 1945 zu beginnen habe und innerhalb von zwei Monaten beendet sein müsse.

Eine eingehende Betrachtung der Pflichtentlassungsliste, die das Herzstück der Richtlinien bildete, zeigt, zu welch grotesken Mitteln die französische Militärregierung gegriffen hatte, um den Eindruck zu erwecken, auch in ihrem Besatzungsgebiet nähere man sich dem amerikanischen Säuberungsstandard: Die umfangreiche Entlassungsliste war nämlich ein reines Schaustück und in der Praxis nicht zu gebrauchen. Die einzelnen Tabellen, aus denen sich die Liste zusammensetzte, hatten die französischen Behörden kurzerhand dem Kapitel über die „Eradication of Nazism“ im SHAEFHandbuch entnommen, hatten sie ohne die geringste Veränderung ${ }^{29}$ einfach aneinandergereiht und in dieser Form ins Zentrum der Säuberungsrichtlinien vom Oktober gestellt. Da diese Tabellen im ,Handbook` aber überwiegend gar keine Pflichtentlas-

${ }^{28}$ Versetzung, Zurückstufung in der Gehaltsklasse bzw. dem Dienstalter, Pensionierung, Entlassung ohne Bezüge.

${ }^{29}$ Die Tabellen sind bis in einzelne Ziffern hinein identisch. Im Erlaß der Milreg findet sich auf der letzten Seite ein Verweis auf Seite 1 des gleichen Erlasses; er verweist auf Kategorie A der Tabelle A. Der französische Redakteur der Note hatte aber auf Seite 1 dies groß A in klein a abgeändert, aber vergessen diese Änderung auf der letzten Seite seines Erlasses anzugleichen. Der deutsche Empfänger der Richtlinien, der das groß A von der letzten Seite für einen Druckfehler halten mußte, hat dann auch prompt in klein a verbessert. In Wirklichkeit stammte groß A aus den amerikanischen Tabellen. 
sungslisten waren und dort in ganz anderem Sinnzusammenhang verwendet waren, war die französische Belastungsliste, die nun den deutschen Stellen zuging, in sich unstimmig, widersprüchlich und in der vorliegenden Form überhaupt nicht anwendbar. Dafür besaß sie aber einen willkommenen Vorteil: sie wies ebenso wie die amerikanische Juli-Direktive über 130 Einzelmerkmale auf und war vorzüglich geeignet, eine drastische Verschärfung der politischen Säuberung in der französischen Zone vorzutäuschen ${ }^{30}$.

Nach dem Wortlaut der Richtlinien sollten alle Mitglieder der Organisationen, die in Tabelle A, sowie alle Funktionäre, die in den Tabellen B und C aufgeführt waren, ,automatiquement' entlassen werden. Die Tabelle A des SHAEF-Handbuches (identisch mit Tabelle A der französischen Anweisung) war aber gar nicht als Formalbelastungsliste konzipiert, sondern ihr Zweck lag allein darin, den alliierten Militärverwaltungen jene Organisationen und Dienststellen der Partei anzugeben, die bei der Besetzung sofort aufzulösen waren ${ }^{31}$ ! Die Tabelle B (identisch mit der Tabelle B der französischen Richtlinien) im ,Handbook ${ }^{`}$ enthielt jene ,,political officers and civil servants“, die entlassen werden konnten, bzw. mußten ${ }^{32}$. Die Tabelle C im Handbuch gab jene Personengruppe an, die von den Offizieren der Militärverwaltung in Zusammenarbeit mit dem, Counter Intelligence Corps' verhaftet und interniert werden muß$\operatorname{ten}^{33}$. Diese Tabelle fehlte naturgemäß in der französischen Note zur Verwaltungssäuberung, da solche Aufgaben ja Sache der ,Contrôle de Sûreté waren. Eine echte Pflichtentlassungsliste war nur die Tabelle $D$ (identisch mit Tabelle $C$ der französischen Note $^{34}$ ) des SHAEF-Handbuches ${ }^{35}$. In ihr waren diejenigen Stellungen in der NSDAP aufgeführt, ,which are to be regarded as disqualifying if held at any time.“ Das Schlüsseldatum für den Parteibeitritt war der 1. Januar 1933; ansonsten waren von den Entlassungsbestimmungen die Funktionäre der Partei, bzw. ihrer Gliederun-

30 Auch wissenschaftlichen Betrachtungen der französischen Entnazifizierungspolitik blieb diese Tatsache verborgen (Fürstenau, Entnazifizierung, S. 137), was zu Fehlinterpretationen führen mußte.

31 S. ,Handbook', Paragraph 280: „The organizations, which are at once to be dissolved are listed in Table ,A'." Diese reichten von den zentralen Dienststellen der NSDAP bis zu den lokalen Organen sowie von den Gliederungen der Partei bis zu den überwachten Verbänden und Berufsorganisationen.

32 Liste B umfaßte maßgebende Beamtengruppen vom Reichsminister bis zum Landrat. Von den 18 Gruppen waren in den amerikanischen Tabellen nur fünf mit einem Sternchen versehen, was bedeutete, daß nur diese entlassen werden mußten.

${ }^{33}$ Sie enthielt u. a. Gestapo-, SD-Mitglieder, Höhere SS- und Polizeiführer u. ä.; ,Handbook', Paragraph 285.

34 Der merkwürdige Umstand, daß eine Tabelle D im französischen Erlaß fehlte, erklärt sich einfach: Die französischen Tabellen A und B sind mit den amerikanischen identisch. Die amerikanische Tabelle C ist im französischen Erlaß weggelassen, denn sie betraf ja nur die zu Internierenden. Die amerikanische Tabelle $\mathrm{D}$ wurde von den Franzosen einfach in Tabelle $\mathrm{C}$ umbenannt. Auch die amerikanische Tabelle E erscheint im französischen Erlaß, sie ist aber aus Versehen nicht (in D) umbenannt worden, so daß im französischen Erlaß eine Tabelle D ganz fehlte.

35 Paragraph 288 des SHAEF-Handbuches: „A list of those Nazi positions, which automatically entail the suspension from office of the holder are shown in Table , $\mathrm{D}$ “". 
gen und die „Alten Kämpfer“ betroffen. An diesen Bestimmungen hatten sich die Richtlinien orientiert, die von den Franzosen während des Stuttgarter Interims herausgegeben worden waren. Nicht einmal redaktionell war diese Liste mit Liste A der französischen Entlassungsbestimmungen koordiniert, denn sie verwies auf einige Parteiorganisationen in Liste A und bestimmte deren Funktionäre zur Entlassung aus dem öffentlichen Dienst. Dies war nun ein Widerspruch in sich, denn schließlich bestimmte die Direktive ja, daß alle Mitglieder der in Liste A aufgeführten Parteiorganisationen zu entlassen seien.

Der erste Eindruck im Staatssekretariat, dem diese Bestimmungen nun vorlagen, war niederschmetternd gewesen. Denn obgleich das Säuberungsverfahren weitgehend in deutschen Händen liegen sollte und auch das kritische Datum für den Parteibeitritt nicht wie in der amerikanischen Zone auf das Jahr 1937 vorgezogen war, konnte der Umschwung, der sich hier in der Entnazifizierung anzukündigen schien, nicht radikaler sein. Schon die ersten Gespräche mit den Militärbehörden über die neuen Anordnungen würden erweisen, ob nun ebenso heiß gegessen werden mußte, was so heiß serviert worden war. Noch am Tage seiner Zuleitung wurde der EntnazifizierungsErlaß in der Direktoriumssitzung durchgesprochen und der Innendirektion zur federführenden Behandlung übergeben. Das Direktorium faßte den Beschluß, „,mit der Militärregierung über die Auslegung gewisser Bestimmungen und damit über eine evtl. Milderung“" zu verhandeln ${ }^{36}$. Damit und mit der Klärung ,in der Note enthaltener Widersprüche“, wurde Staatsrat Schmid beauftragt ${ }^{37}$.

Das Ergebnis seiner Verhandlungen mit dem Gouvernement über die Entschärfung der Direktive dürfte im Staatssekretariat als sensationell empfunden worden sein, denn die Militärregierung zögerte nicht, binnen einer Woche ihre eigenen Anordnungen zu desavouieren. Sie nahm die gerade erlassenen Säuberungsrichtlinien in wesentlichen Punkten wieder zurück und billigte damit die Durchführung der Entnazifizierung nach einem Modus, der mit den Bestimmungen vom 19. Oktober nichts mehr gemein hatte. Die Zugeständnisse der Militärregierung ${ }^{38}$ flossen in den grundlegenden Erlaß der Innendirektion vom 31.10.1945 mit ein ${ }^{39}$, der den deutschen Stellen in Südwürttemberg den neuen Säuberungsmodus bekannt machte.

Das Hauptresultat der Gespräche mit der Besatzungsmacht bestand in der Aushöhlung des Instituts der automatischen Entlassung, denn nun konnten alle Fälle einer individuellen Prüfung unterzogen werden, oder, wie es dazu im Erlaß der Innendirektion in klassischer contradictio in adiecto hieß, die KRUAS hätten zu erwägen, „ob die automatische Entlassung angebracht erscheint oder nicht". Damit war das Kernstück der französischen Anweisung außer Kraft gesetzt, denn ursprünglich hatten die KRUAS ja jeden, auf den eine Formalbelastung zutraf, ,ohne weiteres auszuschließen." Auch bezüglich jener Entlassungen, die von örtlichen Dienststellen der Militärregierung seit der Besetzung ausgesprochen worden waren, erfuhr die Note der Mili-

\footnotetext{
${ }^{36}$ DirS 19. 10. 1945.

37 DirS 23. 10. 1945.

${ }^{38}$ Vergl. den Bericht Carlo Schmids über die Besprechung bei der Milreg; DirS 26. 10. 1945.

39 Erlaß der LdI an alle Landratsämter, Nr. I T 105; SIG, Wü 13/154.
} 
tärregierung eine Abschwächung. Nach ihrem Wortlaut hatten die KRUAS diese Entlassungen lediglich zu bestätigen. Eine Verwendung in anderen Verwaltungszweigen war verboten. Jetzt war es den KRUAS gestattet, ,,in geeigneten Fällen eine Wiederverwendung an anderem Ort zu empfehlen " ${ }^{\text {"40 }}$. Eine gewisse Verschärfung gegenüber den Militärregierungs-Richtlinien bedeutete die Bestimmung, daß jetzt nicht nur die Mitglieder der Tübinger Säuberungskommissionen, sondern auch die KRUA-Mitglieder aus Kreisen ausgewählt werden mußten, die unter dem Nationalsozialismus gelitten hatten. Den KRUAS wuchs nun auch ein Vorschlagsrecht zu. Sie konnten, was in den französischen Bestimmungen nicht vorgesehen war, den Säuberungskommissionen in Tübingen jetzt abgestufte Sanktionsvorschläge unterbreiten. Dieses Vorschlagsrecht, das sich hier eher beiläufig eingeschlichen hatte, sollte sich noch zu einer nicht versiegenden Quelle des Konflikts auswachsen. Entlassungen suchte der LdI-Erlaß den KRUAS möglichst auszureden: „Es besteht kein Bedenken dagegen, bei der als Maßnahme vorgesehenen Entlassung ohne Bezüge in geeigneten Fällen auch eine vorübergehende Außerdienststellung ins Auge zu fassen und dabei die Dauer einer bereits bestehenden Suspendierung zu berücksichtigen." Der Säuberungserlaß der Innendirektion fand die Billigung der Militärregierung ${ }^{41}$ und wurde den Landräten auf der ersten Tagung der Landräte Südwürttembergs mit dem Staatssekretariat in Tübingen übergeben. Damit hatte sich das Gouvernement Militaire, ohne die offiziellen Richtlinien antasten zu müssen, von seinen eigenen Vorschriften distanziert.

Der LdI-Erlaß rief mit seinen schlecht koordinierten und widersprüchlichen Bestimmungen einige Verwirrung hervor. Von den Behörden aus allen Teilen des Landes kamen zahlreiche Rückfragen an das Staatssekretariat ${ }^{42}$. Sie betrafen in der Hauptsache die zweideutigen Entlassungsvorschriften. Einige trafen mit ihrer Auffassung, daß wohl ,an sich automatisch zu Entlassende ... statt für die Eliminierung, für eine gelindere Sanktion vorgeschlagen werden “ könnten ${ }^{43}$, durchaus den Tenor der veränderten Säuberungsbestimmungen. Mündliche Zusagen der Militärregierung bekräftigten diese verblüffende Wende noch einmal. So konnte etwa die Landesdirektion der Justiz dem Landgericht in Hechingen mitteilen, es werde vom Gouvernement Militaire ,überhaupt nicht beanstandet, wenn ... von der automatischen Ausschließung kein Gebrauch gemacht wird“44.

Der deutschen Auftragsverwaltung mögen sich die Beweggründe für die rasche Abkehr der Militärregierung von der Oktober-Direktive nicht ohne weiteres erschlossen haben, doch man erkannte, daß dadurch die Übernahme der radikalen Personalsäuberung amerikanischen Zuschnitts zwar vorgetäuscht, in Wahrheit aber bewußt vermie-

40 Ebd. $\quad 41$ DirS 2.11.1945.

42 S. z. B. die Anfragen des Landgerichtes Hechingen v. 22. 11. 1945 und 28. 11. 1945; StAL, Akten der LdJ, 2014-1/3. Ebenso das Schreiben des Landgerichtes Rottweil v. 12. 12. 1945 (ebd., 20141/9) und das Schreiben des Amtsgerichtes in Freudenstadt an LdJ v. 6. 12. 1945 (ebd., 2014-1/7).

43 Justizamtmann Anton Traber (der spätere Staatskommissar für die politische Säuberung 1947 1948), Hechingen, an die LdJ v. 28. 11. 1945; ebd., Akten der LdJ, 2014-1/3.

44 Schreiben der LdJ an das Landgericht Hechingen v. 11. 12. 1945; ebd., Akten der LdJ 2014-1/4. 
den wurde. Es wurde auch deutlich, daß es das Gouvernement Militaire, das ansonsten bei der Kontrolle der deutschen Verwaltung scharfe Maßstäbe anlegte, in der Entnazifizierung mit den Vorschriften offenkundig nicht sonderlich genau nahm. Dieser Eindruck, den das Staatssekretariat nach einem so aufschlußreichen Intermezzo gewinnen mußte, ließ Raum genug, an der Ernsthaftigkeit der französischen Säuberungsanstrengungen zu zweifeln. Das Gouvernement Militaire verlor damit nicht nur an Glaubwürdigkeit, sondern versetzte der politischen Säuberung schon einen Stoß, bevor sie überhaupt begonnen hatte. Es trug deshalb selbst ein Gutteil der Verantwortung für die laxe Handhabung der Entnazifizierung und die unbrauchbaren Ergebnisse, die dann von den Ausschüssen vorgelegt wurden.

\section{Der Aufbau des Säuberungsapparates}

Der Säuberungserlaß der Innendirektion wurde auf der Tübinger Landrätetagung ausführlich durchgesprochen ${ }^{45}$. Carlo Schmid hob, mit Blick auf die unverändert radikale Säuberungspraxis der Amerikaner in Nordwürttemberg, hervor, ,man müsse tatsächlich der französischen Militärregierung für ihr Verständnis in der Frage des Verwaltungsapparates und dessen Säuberung Dank wissen." Sie verfolge nämlich nicht die Tendenz, den Verwaltungsapparat lahmzulegen. „Wir selbst“, meinte er weiter, sind „,bei dieser Säuberungsaktion nicht als Büttel, sondern als politische Ärzte anzusehen." Er bat deshalb darum, die Militärregierung nicht durch das Überziehen der gesetzten Fristen zu verärgern. Unmittelbar nach der Tagung gingen die ersten Namenslisten für die Besetzung der KRUAS aus den Kreisen ein. Am 10. November waren die Listen aller siebzehn Kreise bis auf eine Ausnahme an die LdI abgesandt. Die Vorschläge waren sehr unterschiedlich. Manche entsprachen völlig dem LdI-Erlaß vom 31. 10. 1945 und stellten in erster Linie NS-Opfer und durch den Nationalsozia-

45 1. Lrtg in Tübingen am 3.11.1945, S. $10 \mathrm{f}$. Die Landrätetagungen in Südwürttemberg waren in der frühen Besatzungszeit eine wichtige politische Institution (dazu Eschenburg, Anfänge, S. 264; Konstanzer, Entstehung, S. 40). In den - später gedruckten - Protokollen mußten allerdings ,mit Rücksicht auf die französische Zensur" (Eschenburg, Anfänge, S. 271) Streichungen vorgenommen werden. Sie sind mindest so aufschlußreich wie der offizielle Text. Einige Beispiele (gestrichene Passagen in Klammern): Carlo Schmid über die Situation der Militärregierung: „Ihre Regierungsmacht ist theoretisch unbeschränkt, praktisch hat sie ihre Schranken (und ich glaube, daß ihre Schranken immer enger werden). “ 7. Lrtg in Saulgau am 4. 5. 1946, S. 35.

Präsident Weiß vom Landesernährungsamt über die Kontroversen um die Lebensmittelablieferung: „Ich versuche immer immer wieder die Franzosen davon zu überzeugen, daß wir einander zu verstehen suchen müssen (,daß wir aber nicht gewillt sind, uns immer mit Einsperren drohen und behandeln zu lassen)." 9. Lrtg in Saulgau am 6. 7.1946, S. 58.

Carlo Schmid zur Erweiterung der deutschen Zuständigkeiten: „In der Presse kann man lesen, daß immer größere Bereiche an die deutsche Verwaltung übergeben werden. Selbständig handeln aber können wir trotzdem nicht (Es hat sich nichts geändert. Nach wie vor gibt man uns Anweisungen. Man gestattet uns zwar einige Worte zu sagen, aber es ändert sich nichts)." 12 . Lrtg in Zwiefalten am 5.10.1946, S. 38. 
lismus Benachteiligte auf ${ }^{46}$, manche Landräte hatten die politische Zusammensetzung in den Vordergrund gestellt ${ }^{47}$, manche gaben keine Schädigung durch den Nationalsozialismus $\mathrm{an}^{48}$; einige Landräte schlugen den Vorsitzenden und die ständigen Mitglieder des KRUA gleich selbst vor ${ }^{49}$, einige gaben keine Präferenzen ${ }^{50}$. Auf alle Fälle fiel dem Landrat in diesem Selektionsprozeß eine Schlüsselrolle zu. Wen er nicht vorschlug, der konnte von vorneherein keinen Sitz in den KRUAS bekommen. Die Beschwerde einer NS-Verfolgten beim Staatssekretariat illustriert die starke Position des Landrates bei der Kandidatenaufstellung. Sie beklagte, daß sich in der örtlichen Hierarchie seit dem „Dritten Reich“ kaum etwas geändert hätte. Die Aussicht auf einen Wandel sei gering, denn ,die Beiräte [KRUA-Mitglieder] wurden vom Landrat diktatorisch ausgesucht. Lauter Männer, die nie wagen würden, gegen einen Beschluß des Landrates aufzutreten ${ }^{* 51}$. Wie die Analyse der Listen ergibt, änderte sich an der einmal von den Landräten vorgeschlagenen Zusammensetzung praktisch nichts mehr. Das Staatssekretariat, das aus den zwölf bis fünfzehn Kandidaten fünf ständige Mitglieder und drei Ersatzleute auswählen mußte, hat in keinem einzigen Fall die Vorschläge eines Landrates als ungenügend oder einseitig beanstandet oder gar eine Neuaufstellung verlangt.

Die Auswahl der Kandidaten zur Vorlage bei der Militärregierung geschah vor allem nach technischen Gesichtspunkten, d. h. die LdI schied in der Regel die Beamten aus, die sie gleich als die Fachbeisitzer für die Innenverwaltung nominieren konnte ${ }^{52}$. Die LdI akzeptierte in der Regel auch die Vorschläge der Landräte für die fünf ständigen Vertreter ${ }^{53}$, den Vorsitzenden und dessen Stellvertreter ${ }^{54}$. Abgelehnt wurden Ortsfremde $^{55}$ und Kandidaten, die das 70 . Lebensjahr schon überschritten hatten ${ }^{56}$ sowie Bürgermeister und Landräte, die sich da und dort selbst vorgeschlagen hatten ${ }^{57}$. Katholischen Geistlichen war die Úbernahme dieses Amtes von Seiten der Kirche nicht gestattet $^{58}$. Eine Verlagerung der in den Vorschlagslisten zum Ausdruck gekommenen politischen Gewichtsverteilung nahm die Innendirektion nicht mehr vor. Von zwei Landkreisen abgesehen, gingen die endgültigen Nominierungslisten aller KRUAS am 19. November 1945 zur Genehmigung an die Militärregierung.

${ }^{46}$ So der KRUA in Reutlingen (SIG, Wü 13/154 und Wü 13/167), wo die Hälfte der Kandidaten im $\mathrm{KZ}$ gesessen hatte. Alle waren laut Oskar Kalbfell „durch das nationalsozialistische Regime geschädigt."

47 In Tettnang, wo es in der Vorschlagsliste hieß: „Die politische Zusammensetzung entspricht auch den Wahlergebnissen vom 6.11.1932 und 5.3.1933 ... unter Weglassung der NSDAP." SIG, Wü 13/154 und Wü 13/171.

48 Saulgau. Alle Kreise in: SIG, Wü 13/158-174.

49 Biberach, Reutlingen, Calw.

so Ehingen, Horb.

${ }^{51}$ Schreiben einer Ärztin v. 19. 1. 1946; SIG, Wü 42P, acc. 10/1970, A 13150.

s2 Calw; SIG, Wü 13/160. Tübingen; SIG, Wü 13/172.

${ }^{53}$ Ravensburg; SIG, Wü 13/166 und 154.

54 Biberach; SIG, Wü 13/159 und 154.

55 Ebd.

56 Sigmaringen; SIG, Wü 13/170 und 154. Tettnang; SIG, Wü 13/171.

57 Freudenstadt; SIG, Wü 13/162 und 154. $\quad 58$ Tettnang; wie Anm. 56. 
Entscheidenden Einfluß auf die Zusammensetzung der Säuberungsgremien hatten theoretisch die Besatzungsbehörden, doch praktisch machten sie ihn nicht geltend. Sie akzeptierten 93 Prozent der KRUA-Mitglieder und auch die Zusammensetzung der Ausschüsse ohne Einspruch. Die Militärregierung hatte dem Staatssekretariat bei der Aufstellung des Säuberungsapparates praktisch freie Hand gelassen. Eine Analyse der personellen Struktur der Ausschüsse zeigt aber, daß nur gut die Hälfte der Mitglieder die vorgeschriebenen politischen oder persönlichen Voraussetzungen für einen Sitz in den Säuberungsausschüssen mitbrachte. Bei der Bestimmung der Struktur der Ausschüsse ist man auf die Angaben der Landräte in den Vorschlagslisten angewiesen, doch selbst die Anlegung wohlwollender Maßstäbe ergibt, daß die Forderung des LdIErlasses, nach der die KRUA-Mitglieder ,persönlich, beruflich oder in ihrem Vermögen durch das nat. soz. Regime geschädigt" sein mußten, vom KRUA-Personal in seiner Gesamtheit nicht annähernd erfüllt wurde: Maximal 27 Prozent waren NSVerfolgte, höchstens weitere 31 Prozent waren beruflich ernstlich behindert gewesen $^{59}$. Die Nachlässigkeit bei der Aufstellung des Apparates sollte sich bald in der Qualität seiner Arbeit widerspiegeln. Die unterschiedliche personelle Struktur der KRUAS mußte in Anbetracht der verschwommenen Richtlinien eine untragbar ungleiche Beurteilung gleichartiger Fälle zur Folge haben, die auch von der zweiten Säuberungsinstanz, den Säuberungskommissionen, nicht mehr zu bereinigen war.

Die Militärregierung hatte mit ihrer Terminsetzung für den Abschluß des Säuberungsverfahrens zum Jahresende 1945 den beträchtlichen Personal- und Verwaltungsaufwand, der zum Aufbau des Entnazifizierungsapparates erforderlich war, bei weitem unterschätzt. $\mathrm{Zu}$ diesem Schlußtermin hatte in Südwürttemberg die Entnazifizierung noch nicht einmal begonnen: Ende Dezember stand lediglich die Zusammensetzung der KRUAS fest. Aber die Ausschüsse konnten ihre Arbeit auch jetzt noch nicht aufnehmen, da bis zum Jahreswechsel ein großer Teil der Beamtenbeisitzer nicht mehr ernannt werden konnte ${ }^{60}$. Es zeichneten sich allmählich personelle Grenzen ab, denn

$59 \mathrm{Zu}$ den NS-Verfolgten sind alle jene KRUA-Mitglieder gerechnet, die laut Angaben des Landrates mehrere Monate/Jahre aus politischen Gründen im KZ/Gefängnis gesessen hatten. In der zweiten Gruppe sind all jene zusammengefaßt, die mit klaren Sanktionen, wie Berufsverbot oder Entlassung, belegt gewesen waren. Nicht mitgerechnet sind jene, bei denen die Angaben allzu vage bleiben, wie „,wurde immer kritisiert" oder „Konflikte mit den Nazis“ u. ä. Sie scheinen die LdIKriterien nicht zu erfüllen, zumal die Landräte das Licht ihrer Kandidaten nicht unter den Scheffel gestellt haben dürften. 34 Prozent der Mitglieder waren ehemalige Zentrumsanhänger gewesen, 23 Prozent standen der SPD nahe, 14 Prozent der KPD und 10 Prozent den liberalen Parteien; 19 Prozent waren ohne Parteipräferenz. Das Durchschnittsalter der Ausschüsse lag zwischen 50 und 60 Jahren; allein 30 Prozent der ständigen Mitglieder wurde von Beamten gestellt.

${ }^{60}$ Für die 15 Verwaltungsgliederungen der sechs Landesdirektionen waren insgesamt 765 Beisitzer aus der Beamtenschaft zu ernennen. Die Beisitzer der bedeutenden Gruppe „Innenverwaltung“ waren, von zwei Kreisen abgesehen, am 23.1.1946 ernannt worden (SIG, Wü 13/154), die Beisitzer für die Lehrerschaft am 6. 2. 1946 (SIG, Wü 13/130) und die Vertreter der Justiz gar erst am 4.5.1946 (StAL, Akten der LdJ, 2014-3/24). Die Ursache für die Verzögerungen lag zum Teil bei der Milreg selbst. Sie ließ sich zur Genehmigung von Vorschlägen oft mehr als einen Monat Zeit. Auch ganz praktische Schwierigkeiten waren im Spiel. Der Beisitzer der Gesundheits- 
das Reservoir politisch unbelasteter Beamter war nicht unerschöpflich. Staatsrat Schmid, der in dieser Zeit häufig an den Stuttgarter Kabinettssitzungen teilnahm, wies auf die Probleme der Personalrekrutierung hin. Er berichtete dort anläßlich der Diskussion um die Einführung des Spruchkammerverfahrens, ,,daß es schon in der französischen Zone schwierig war, die zur Durchführung der Entnazifizierung erforderlichen Leute zu finden" ${ }^{\text {"61. }}$. Inzwischen drängte die Militärregierung wiederholt darauf, daß wenigstens die schon bestehenden KRUAS ihre Arbeit aufnehmen sollten ${ }^{62}$. Auch der Innendirektion war daran gelegen, daß die Ausschüsse zum frühest möglichen Zeitpunkt mit der Überprüfung der Beamtenschaft begannen, ,,um die auf den Dienstbetrieb lähmend wirkende Ungewißheit der Weiterverwendung oder Entlassung baldmöglichst auszuschalten "63. Sie ordnete deshalb für ihren Bereich die amtsinterne Erfassung allen Belastungsmaterials an, das den KRUAS sofort nach deren Konstituierung zur Verfügung gestellt werden sollte. Diese unterstützenden Maßnahmen konnten allerdings erst dann greifen, wenn in den Landkreisen arbeitsfähige KRUAS existierten. Die Mehrzahl konnte ihre Arbeit aber erst zu Beginn des Jahres 1946 aufnehmen. Endgültige Säuberungsentscheidungen, die nur durch die Säuberungskommissionen bei den Landesdirektionen getroffen werden konnten, waren demnach vor Jahresende ohnehin nicht mehr zu erwarten ${ }^{64}$. Doch Anfang 1946 war offenbar ein Punkt erreicht, wo das Gouvernement Militaire nicht mehr länger auf ein vorweisbares Ergebnis bei der Entnazifizierung seiner Zone verzichten konnte.

\section{Erste Resultate}

Mehr als nur gute Vorsätze für das neue Jahr standen hinter der harten Forderung der Militärregierung zu Beginn des Jahres 1946, die Säuberung der Verwaltung müsse „unter allen Umständen“ 65 bis Ende Januar beendet sein. Diese ebenso unrealistische wie unvermittelte Anordnung (immerhin hatte sich das Gouvernement Militaire zur Genehmigung der Personalvorschläge für die Säuberungsgremien selbst wochenlang Zeit gelassen) entsprang nicht den gleichsam über Nacht erwachten prinzipiellen Bedenken der französischen Militärbehörden gegenüber der nach wie vor gänzlich unbefriedigenden Entnazifizierung in ihrem Besatzungsgebiet, sondern diese plötzli-

verwaltung im Kreis Wangen stellte seine Mitarbeit in einem Schreiben an die LdI (17. 1. 1946) für die Zeit in Frage, ,solange die Benzinversorgung so schlecht ist.“ SIG, Wü 13/329.

${ }^{61}$ Kabinettssitzung des StaMi in Stuttgart am 19.2. 1946. HStASt EA 1/20, C 1/32. Zu dieser Sitzung s. a. Niethammer, Entnazifizierung, S. 323.

62 Schreiben der Milreg an das Staatssekretariat v. 6.12.1945; SIG, Wü 13/239.

${ }^{63}$ Erlaß der LdI an alle Polizeidienststellen v. 17.11.1945; SIG, Wü 13/158.

${ }^{64}$ Hierzu die Kritik des Parlamentarischen Untersuchungsausschusses der französischen Verfassunggebenden Nationalversammlung: „La plupart des commissions d'épuration et des délégations d'instructions n'ont commencé à travailler qu'à l'arrivée de la commission parlamentaire d'enquête [Februar 1946], soit près de quatre mois après leur institution par l'administrateur général." Assemblée, Documents, Annexe Nr. 924.

65 GouvS 8. 1. 1946. 
che Forcierung erklärt sich aus dem Umstand, daß sich in Paris gerade eine Parlamentarische Untersuchungskommission der Verfassunggebenden Nationalversammlung konstituiert hatte ${ }^{66}$, mit deren Eintreffen in der französischen Zone in den ersten Wochen des Jahres gerechnet werden mußte. Da diese in Südwürttemberg alsbald feststellen würde, daß die systematische politische Überprüfung, die eigentlich schon hätte abgeschlossen sein müssen, noch nicht einmal begonnen hatte, entfaltete das Gouvernement Militaire eine geradezu hektische Aktivität bei dem Versuch, in aller Eile einige augenfällige Mängel des Säuberungsverfahrens zu korrigieren und doch noch etwas Zahlenmaterial zur Entnazifizierung vorlegen zu können. Es mag sein, daß zusätzlich auch noch einer der alliierten Vertreter im Kontrollrat, wo die Direktive Nr. 24 gerade kurz vor der Verabschiedung stand, wieder einmal auf den desolaten Stand der Entnazifizierung in der französischen Zone hingewiesen und die französische Delegation an die Grundsätze des Potsdamer Abkommens über die Beseitigung des Nationalsozialismus in Deutschland erinnert hatte, die auch von Frankreich anerkannt worden waren ${ }^{67}$. In diesem Zusammenhang überliefert jedenfalls Gebhard Müller, er wisse aus Gesprächen mit Justizoffizieren der französischen Militärregierung in Tübingen, daß ,die Amerikaner, vor allem aber die Russen, immer wieder beanstandet haben, daß die Entnazifizierung in der französischen Zone nicht hart genug durchgeführt werde" ${ }^{68}$. Das Gouvernement Militaire zog aus dem gestiegenen Erwartungsdruck erst einmal organisatorische Konsequenzen und betraute nun formell einen ihrer Offiziere mit dem Amt des Entnazifizierungskommissars ${ }^{69}$. Auch im Staatssekretariat, wo bisher die Innendirektion die Organisation des Entnazifizierungsverfahrens mitbesorgt hatte, legte man der Durchführung der politischen Säuberung durch die Ernennung des Chefs des Direktorialamtes ${ }^{70}$, G. H. Müller ${ }^{71}$, zum Säuberungskommissar ein größeres Gewicht bei ${ }^{72}$. Müller begann seine Tätigkeit als Koordinator der politischen Säuberung der Verwaltung mit einem dringenden Telegramm an alle Landräte: „Auf Befehl der Militärregierung ist die Entnazifizierung der Verwaltung äußerst zu beschleunigen. Arbeit der Untersuchungsausschüsse mit allen Mitteln vorantreiben. Letzte Ergebnisse müssen bis spätestens 25. Januar hier eintreffen. Notfalls Kurier schicken"73. Selbst die noch im Aufbau befindlichen KRUAS waren angewiesen, sofort mit ihrer Tätigkeit zu beginnen und laufend über Art und Umfang der ausgesprochenen Sanktionen zu berichten. Zu gleicher Zeit bemühte sich

66 Vergl. Henke, Aspekte, S. $183 \mathrm{f}$.

67 Schreiben des Außenministers der Provisorischen Französischen Regierung an die Botschafter der Vereinigten Staaten, des Vereinigten Königreiches und der Sowjetunion v. 7. 8. 1945, in: Französische Dokumente über Deutschland, Nr. II, Europa-Archiv v. 20. 8. 1954, S. 6745. Dazu s. a. Abendroth, Frankreich, S. 76.

68 Information Gebhard Müller.

${ }^{69}$ Hauptmann Vigouroux. GouvS 8. 1. 1946.

70 Das Direktorialamt bestand seit dem 1.2. 1946. Vergl. Konstanzer, Entstehung, S. 33.

71 G. H. Müller (SPD) ist nicht zu verwechseln mit Dr. Gebhard Müller (CDU), dem späteren Staatspräsidenten von Württemberg-Hohenzollern, der damals in der LdJ tätig war.

72 DirS 8. 1. 1946 und 11. 1. 1946.

73 Telegramm v. 11. 1. 1946; SIG, Wü $15 / 655$. 
die Militärregierung in aller Eile, einen prinzipiellen Mangel des Säuberungsverfahrens zu beheben. Denn bisher waren nur Beamte und Angestellte des öffentlichen Dienstes und ein bestimmter Teil in der freien Wirtschaft Tätiger betroffen; Freiberufliche unterlagen dem Verfahren noch nicht. Das Gouvernement Militaire erweiterte nun ziemlich planlos den Betroffenenkreis. So sollten jetzt auch die Rechtsanwälte ${ }^{74}$ und die Ärzteschaft ${ }^{75}$ der politischen Überprüfung unterzogen werden." Selbst Schornsteinfeger ${ }^{76}$ und Feuerwehrleute ${ }^{77}$ wurden jetzt pauschal einbezogen. Genügend andere Berufszweige, die von der politischen Säuberung ebenfalls noch nicht betroffen waren, blieben von den neuen Anordnungen ausgenommen.

Am 21. Januar 1946 lagen dem Staatssekretariat die angeforderten Zwischenresultate der politischen Ưberprüfung vor; sie waren quantitativ wie qualitativ höchst unterschiedlich ausgefallen. Bislang war in Südwürttemberg etwa ein Drittel der Beamtenschaft überprüft worden ${ }^{78}$. Manche KRUAS waren in ihrer Arbeit bereits weit fortgeschritten, andere hatten noch so gut wie keine Ergebnisse vorzuweisen ${ }^{79}$. Insgesamt sollten nach den Vorschlägen der KRUAS 78 Prozent aller Überprüften ohne Sanktionen im Amt belassen werden, 2 Prozent waren zur Versetzung, gut 1,5 Prozent zur Pensionierung vorgeschlagen; rund 11,0 Prozent wurden zur Zurückversetzung, 7,5 Prozent zur Entlassung ohne Bezüge vorgesehen. Bleiben die Gesamtzahlen für sich gesehen ziemlich aussagearm, so zeigt die Einzelanalyse die enormen Unterschiede in der Arbeitsweise der KRUAS: Die Ergebnisse der Überprüfungen klafften von Kreis zu Kreis himmelweit auseinander. So betrug etwa in einem Kreis der Prozentsatz der Sanktionierten 57 Prozent, in einem anderen dagegen nur 8 Prozent $^{80}$. In manchen Kreisen hatten die KRUAS prozentual gut dreimal soviele Beamte zur Entlassung vorgeschlagen wie anderswo ${ }^{81}$. In den Kreisen Wangen und Horb wollte man 1 Prozent der Úberprüften in ihrem Besoldungsdienstalter zurückstufen, in Hechingen und Balingen 40 Prozent. Von Pensionierungen und Versetzungen wurde nur wenig Gebrauch gemacht, da diese Sanktionen die Staatskasse zusätzlich belastet hätten. Es ist anzunehmen, daß die Landesdirektion der Finanzen dies den KRUAS schon vorab ans Herz gelegt hatte, noch ehe sie den entsprechenden Erlaß herausgab ${ }^{82}$. Darin wurden die KRUAS gebeten, nicht zu viele Versetzungen vorzunehmen, denn es sei „zu befürchten, daß die Zahl der Beamten, an die Trennungsentschädigung geleistet

${ }^{74}$ Schreiben der LdJ an alle Justizbehörden v. 18. 1. 1946; SIG, Wü 13/77.

75 GouvS 22. 1. 1946.

${ }^{76}$ Erlaß der LdJ an alle Landratsämter v. 10.1. 1946; SIG, Wü 15/513.

77 Desgl. v. 4. 2. 1946; ebd.

78 Die folgenden Berechnungen beruhen auf den Ergebnismeldungen der KRUAS in: SIG, Wü 13/ 238. Insgesamt waren 10883 Fälle bearbeitet.

79 In Rottweil waren die Akten schon abgeschlossen, in Tuttlingen waren erst 4 Prozent, in Freudenstadt gar erst 2,5 Prozent der zu prüfenden Fälle bearbeitet.

8052 Prozent irn Kreis Balingen, 8 Prozent im Kreis Wangen. Bei der Berechnung der prozentualen Verteilung der Sanktionen wurden nur die zwölf Kreise herangezogen, bei denen die Zahl der insgesamt Uberprüften mehr als 300 Personen betrug. (Ausnahme Horb: 230 Fälle).

81 In Calw 3,5 Prozent; in Reutlingen, Saulgau, Balingen 12 Prozent.

${ }^{82}$ Schreiben der LdF an alle KRUAS v. 26. 1. 1946; SIG, Wü 15/77. 
werden muß, dabei im erheblichen Umfange steigt, wodurch eine nicht unbeträchtliche finanzielle Belastung für den Staat eintritt.“ Der Landrat in Rottweil erkannte diese Empfehlung als Aufforderung zur Verwässerung der politischen Säuberung durch sachfremde Erwägungen und sah sich deshalb, wie er der Landesdirektion mitteilte, nicht in der Lage, bei dem KRUA in Rottweil eine Änderung der Versetzungsvorschläge anzuregen ${ }^{83}$. Der starke Beamtenanteil unter den ständigen Mitgliedern der KRUAS wirkte sich bei den Entscheidungen nicht in einer bestimmten Richtung aus ${ }^{84}$. Jedoch zeigten die KRUAS, deren Mitglieder unter dem Nationalsozialismus schwere Nachteile erlitten hatten, eine Tendenz zu schärferem Vorgehen als jene, bei denen diese Merkmale fehlten ${ }^{85}$.

Durchgehend verraten die Vorschläge der KRUAS, daß die Ausschüsse - ungeachtet aller erdenklichen Erklärungs- oder Leugnungsversuche der Betroffenen - in ihrem überschaubaren Einzugsbereich gut in der Lage waren, das politische Engagement des einzelnen für den Nationalsozialismus in den zurückliegenden Jahren zu beurteilen. Kriterien formaler Belastung, wie die Mitgliedschaft in NS-Organisationen, Beitrittsdatum u. ä. haben dabei offenbar nur eine untergeordnete Rolle gespielt. In den Begründungen der Sanktionsvorschläge kommt die intime Vertrautheit der Ausschüsse mit dem Einzelfall zum Ausdruck. So etwa - um einige beliebig ausgewählte Beispiele anzuführen - im Falle eines Gastwirts aus einem Dorf bei Reutlingen, der 1933 Mitglied der NSDAP, 1935 SA-Mann geworden, von den formalen Kriterien her also eher ein kleines Licht gewesen war. Gleichwohl schlug der Ausschuß langdauernde empfindliche Arbeitsverpflichtungen vor: „N. forderte durch seine Haltung und Einstellung allgemein das Urteil heraus“, so der Reutlinger Ausschuß in der Begründung seines schwerwiegenden Vorschlages, „daß er ein ,Obernazi“ sei. Sein Gasthaus war die Hochburg der Nationalsozialisten, in dem auch das Führerkorps von Münsingen und Reutlingen verkehrte und sich, durchfraß $\beta^{\prime}$... Das zur Entlastung beigebrachte Zeugnis einiger Polen über gute Behandlung und Unterstützung mit markenfreien Lebensmitteln ist diesen offenbar zur Unterzeichnung vorgelegt worden und wird als nicht glaubwürdig angesehen, ebensowenig die darin enthaltene Angabe, daß ausgerechnet $\mathrm{N}$. als überzeugter Nationalsozialist einen abgesprungenen amerikanischen Piloten acht Tage vor den deutschen Behörden verborgen gehalten haben will.

83 Schreiben an die LdF v. 6. 2. 1946; SIG, Wü 140/77/6000.

${ }^{84}$ Im KRUA Balingen saßen z. B. vier Beamte als ständige Mitglieder; er sprach in 52 Prozent der Fälle Sanktionierungen aus (Addition der Prozentzahlen der beiden hautpsächlich angewandten Sanktionen, „Entlassung“ und „Zurückversetzung“). Im KRUA Wangen war unter den ständigen Mitgliedern ein Beamter; hier wurden in 5 Prozent der Fälle Sanktionen ausgesprochen. Dagegen waren vom KRUA Biberach, in dem kein Beamter saß, immerhin in 29 Prozent der Fälle Sanktionen verhängt worden.

85 Im Balinger KRUA, der in 52 Prozent der Fälle Sanktionen verhängte, saßen zwei ehemalige SPDund zwei ehemalige KPD-Mitglieder. Von sechs der acht Mitglieder hatten fünf im KZ oder Gefängnis gesessen, ein weiterer war 1937 als Schulleiter entlassen worden. Im KRUA Wangen saß kein Vertreter der Arbeiterparteien; nur bei einem einzigen, einem Zahnarzt, war auf eine Benachteiligung während der NS-Zeit hingewiesen. Er sei wegen seiner jüdischen Frau „kritisiert“ worden. Dieser KRUA verhängte in 5 Prozent aller Fälle Sanktionen. 
Von diesem Piloten weiß niemand in [dem Ort] B. etwas.“ Die Berücksichtigung dessen spezifischer Verhältnisse bewog den Ausschuß dazu, bei einem selbständigen Kaufmann mit praktisch der gleichen formalen Belastung die Zahlung der vergleichsweise geringfügigen Geldbuße in Höhe von 2000 RM für angemessen zu halten: „B. hat sich recht aktiv für die Partei eingesetzt und sich auch auf die Stellung seines Schwagers als Kreisleiter in A. etwas zu Gute gehalten. Wenn er später auch - offenbar aus familiären Gründen - in Gegensatz zu diesem kam und deshalb auch Ungelegenheiten hatte, so hat sich doch nach allgemeiner Meinung B. den damaligen Verhältnissen recht gut anzupassen gewußt, ebenso wie dies neuerdings offenbar wieder der Fall ist. " Den Besitzer einer Omnibus-Vermietung, Pg seit 1938 und kleiner Funktionär des NSKK, wollte der Reutlinger KRUA dagegen harte Arbeitsverpflichtung auferlegen sowie dessen Fahrzeugbestand einziehen: „R. war ein gläubiger und unbelehrbarer Nazi, so unbelehrbar, daß er noch jetzt äußert, daß die KZ's zu Recht bestanden hätten und viel zu wenige im $\mathrm{KZ}$ gewesen seien" ${ }^{\text {"86. }}$.

Das insgesamt gesehen unbrauchbare Ergebnis, das in dieser Zwischenbilanz jetzt deutlich wurde, war weitgehend Folge der unterschiedlichen Personalstruktur in den Ausschüssen und der allgemein gehaltenen Säuberungsbestimmungen. Nun rächte sich die Nachlässigkeit, mit der die Landesdirektion des Innern und die französischen Behörden die Aufstellung der KRUAS betrieben hatten. Im Interesse einer beschleunigten Abwicklung der Säuberung war praktisch jede Zusammensetzung der KRUAS, wie sie die Landräte vorschlugen, gebilligt worden - auch wenn sie dem LdI-Erlaß vom Oktober klar widersprach. Damit hatte man das Gegenteil von dem erreicht, was das Ziel der beschleunigten Erledigung der Entnazifizierung gewesen war: Die eklatant voneinander abweichenden Säuberungsergebnisse fachten die allgemeine Unruhe und die politischen Spannungen in der Bevölkerung und den Behörden erst richtig an. Wenn ein Forum zur offenen Diskussion politischer Differenzen unter den Bedingungen der Auftragsverwaltung in Südwürttemberg auch noch fehlte, kamen politische Gegensätze in der Zeit vor Neubildung der Parteien und Parlamentarisierung des Landes in verdeckter Form in den Landrätetagungen dennoch zum Ausdruck. So versuchte beispielsweise der Ravensburger Landrat, dessen Personalpolitik in einer Tagung unter Beschuß geraten war ${ }^{87}$, dieser Kritik mit der Forderung an die KRUAS die Spitze abzubrechen, daß ,auch in den Säuberungsausschüssen noch schärfer vorgegangen wird als bisher." Ein KRUA-Vorsitzender hielt ihm jedoch sogleich entgegen: „Wegen der Entnazifizierung haben mich die Ausführungen von Herrn Landrat B. etwas befremdet, denn wir haben gestern abend erst Klagen über Ravensburg gehört, die mit diesen Ausführungen in Widerspruch stehen. (Redner führt einige Beispiele an.)." Schließlich beendete Innendirektor Roßmann diese Auseinanderset-

86 Zitate aus SIG, Wü 15/315.

${ }^{87}$ Der Oberbürgermeister von Tübingen hatte verlangt, daß „künftig auch im Kreis Ravensburg die Leute ausschließlich aus diesen Kreisen [,wirkliche Demokraten' im Gegensatz zu ehemaligen Nationalsozialisten] zur Mitarbeit beigezogen werden, um auch der Öffentlichkeit zu zeigen, daß diese Dinge nicht auf die lange Bank geschoben werden." 5. Lrtg in Ravensburg am 2. 3.1946, S. 21. 
zung, in der weniger persönliche Animositäten, sondern kontroverse politische Vorstellungen zur Entnazifizierung ans Licht drängten. Er bedauerte, daß die Diskussion auf „das politische Gebiet“ abgeglitten sei: „Wir sind zu einer Arbeitstagung zusammengetreten und zu keiner politischen Versammlung. Alle Anfeindungen in dieser Hinsicht bitte ich, beiseitezulegen. Wir sind dazu da, am selben Strang zu ziehen und zu arbeiten, um die Situation unseres armen Landes zu verbessern und nicht dazu, daß wir uns streiten" ${ }^{\prime 88}$. Diese Auffassung Roßmanns, der hier die Verwaltungstätigkeit mit der Aura des Unpolitisch-Sachlichen umgab, war in der Auftragsverwaltung weit verbreitet. Sie mußte die Untersuchungsausschüsse in einer Sicht der politischen Säuberung bestärken, die Carlo Schmid in einer Bilanz der Arbeit der KRUAS zum Gegenstand seiner Kritik machte: „Wir wollen uns über eines klar sein, es ist auf diesem Gebiet nicht überall so gearbeitet worden, wie es wünschenswert gewesen wäre. Man hat zum Teil dieses Problem der politischen Säuberung ausschließlich unter dem Gesichtspunkt gesehen, inwieweit man durch diese Maßnahmen in seiner Verwaltungstätigkeit gestört wird“899.

Auch die Militärregierung war mit der Arbeit der KRUAS nicht zufrieden. In einigen Kreisen, bemängeite der Gouverneur, sei die Säuberung zu milde gehandhabt wor$\mathrm{den}^{90}$; vor allem aber seien die Stellungnahmen der Ausschüsse in den einzelnen Kreisen ,außerordentlich unterschiedlich ausgefallen“91. Der Gouverneur gab die Sache aber noch nicht verloren und setzte seine Hoffnung nun auf die Säuberungskommissionen in Tübingen. Doch wie sollten es die Kommissionen bewerkstelligen, die enorm divergierenden Säuberungsvorschläge auf einen annähernd gleichen Nenner zu bringen, ohne die ganze Arbeit der Ausschüsse zu desavouieren? Die Kommissionen mußten entweder die meisten Vorschläge wieder aufheben oder die Fälle zur nochmaligen Bearbeitung zurückgeben. Eine solche Brüskierung mußte die KRUAS aber entweder in die Resignation treiben oder ihre Autorität in den Kreisen in einem Maße untergraben, der den Fortgang der Entnazifizierung in erster Instanz ernstlich in Frage stellte.

Nachdem der Militärregierung die Zwischenbilanz der KRUAS vorlag, wandte sich Hauptmann Vigouroux, der Säuberungskommissar des Gouvernement Militaire, an G. H. Müller und forderte eine Prognose über die voraussichtliche Gesamtdauer des Säuberungsverfahrens in Südwürttemberg an. Der Chef des Direktorialamtes deutete in seinem Memorandum wohl vorsichtig die Schwierigkeiten an, die sich für die Säuberungskommissionen aus der unterschiedlichen Arbeitsweise der KRUAS ergaben $^{92}$, legte ansonsten seinen Berechnungen aber ein unrealistisch rasches Arbeitstempo der Kommissionen zugrunde. Bei wirklichkeitsnaher Interpretation des Memorandums mußte die Militärregierung nun davon ausgehen, daß sich das Säube-

88 Ebd., S. 24 f.

89 7. Lrtg in Tettnang am 4. 5. 1946, S. 4.

90 GouvS 1.2. 1946.

${ }^{91}$ GouvS 14. 2. 1946.

92 „... wenn für den Großteil der Fälle tatsächlich keine detaillierte Diskussion erforderlich sein sollte.“ Memorandum von G. H. Müller an die Militärregierung v. 22. 1. 1946; SIG, Wü 13/238. 
rungsverfahren bis in den Sommer hinziehen würde, ein halbes Jahr länger als in der Note vom Oktober angeordnet. Und noch waren die Kommissionen nicht einmal aufgestellt. Im Staatssekretariat hatte man sich um eine rasche Aufstellung der Säuberungskommissionen bemüht. Schon Ende November waren die Personalvorschläge an die Militärregierung abgegangen ${ }^{93}$. Dort ließ man sich zwei Monate mit der Genehmigung Zeit, da die Installierung der Kommissionen solange nutzlos bleiben mußte, wie die KRUAS ihre Arbeit noch nicht aufgenommen hatten ${ }^{94}$. Die personelle Struktur der Kommissionen entsprach wiederum nicht den Vorschriften. Nur etwa die Hälfte der Mitglieder hatte unter dem Nationalsozialismus ,,in ihrer Person, in ihrer Laufbahn oder in ihrem Vermögen" Nachteile irgendeiner Art erlitten. Der Einfluß der Kommunisten in den Säuberungsgremien der zweiten Instanz war ganz ausgeschaltet wor$\mathrm{den}^{95}$; ein Drittel der Kommissionsmitglieder waren Sozialdemokraten. Diese Zusammensetzung war von der Militärregierung ohne Abstriche gebilligt worden.

Ein Teil der Kommissionsmitglieder hatte wenig oder gar kein Interesse an ihrer Mitwirkung bei der Entnazifizierung. Sie versuchten, sich dieser undankbaren Aufgaben zu entziehen. Besonders die freiberuflichen Mitglieder der Kommissionen gaben dem Aufbau ihrer beruflichen Existenz den Vorzug. Sie bedachten wohl auch die möglichen Spätfolgen einer so prononcierten politischen Tätigkeit, die ihre Karriere eines Tages beeinträchtigen konnten ${ }^{96}$. Aber selbst beamtete Kommissionsmitglieder folgten ihrer Ernennung nur widerwillig und unter großem inneren Vorbehalt. So wollte sich der Vorsitzende (!) der Säuberungskommission für die Innen- und Finanzverwaltung, ein Ministerialrat der Innendirektion, ,unter keinen Umständen“" mit seiner Ernennung abfinden. Er mußte sich nach einigem Hin und Her schließlich fügen, nachdem Landesdirektor Roßmann ihm klargemacht hatte, der Gouverneur habe inzwischen angedroht, daß er ,Leute, die sich der Mitgliedschaft im Säuberungsausschuß entziehen wollten, zur Bestrafung bringen werde ${ }^{\text {‘97 }}$. Auch freiberufliche Kommissionsmitglieder wurden von ihrer Mitwirkung nicht ohne weiteres entbunden ${ }^{98}$.

93 Schreiben der LdI an die Milreg v. 29.11.1945; SIG, Wü 13/154.

94 Schreiben der Milreg an die LdI v. 29.1. 1946; SIG, Wü 13/172. Die folgenden Angaben sind dem Schreiben der LdI an die Kommissionsvorsitzenden v. 8.2. 1946 (SIG; Wü 13/154) entnommen.

95 Von den 27 benannten Mitgliedern gehörte eines der KPD an. Dieser Beisitzer war als zweiter Ersatzmann in der Säuberungskommission für die Arbeits- und Wirtschaftsverwaltung vorgesehen. In den KRUAS hatte der Anteil der kommunistischen Mitglieder noch $14 \%$ betragen.

96 So führte ein Tübinger Verlagsinhaber an, ,seit dem Wiederaufbau einer Organisation der Buchhändler laste derart viel auf ihm", daß er seinen Aufgaben in der Kommission nicht nachkommen könne: „Andere, ältere Aufgaben haben die ersten Rechte.“ Ein Anwalt und Notar „bittet von dieser Ernennung Abstand nehmen zu wollen, da ... ich mit dem besten Willen nicht die Zeit für eine derartige Tätigkeit aufbringen kann, zumal mich mein Beruf schon über meine Kräfte in Anspruch nimmt.“ Ein Professor bat ,um Entbindung von diesem Auftrag: Ich habe zur Zeit verschiedene längere und kürzere Reisen vor mir im Hinblick auf meine Berufszukunft." Alles in: SIG, Wü 13/239.

97 Notiz eines Ministerialrats für Landesdirektor Roßmann v. 18.3. 1946; SIG, Wü 13/239.

98 Direktor Roßmann stellte in seinem ablehnenden Bescheid fest: „Für die Überprüfungsarbeiten 
Bevor die Säuberungskommissionen am 18. Februar 1946 erstmals zusammentraten, fand in den Landesdirektionen ein Gedankenaustausch über die Normen statt, mit denen man eine einheitliche Bemessung der Sanktionen sicherstellen wollte. Da man die Haltung der Militärregierung zu ihrer Oktober-Direktive kennengelernt hatte, konnte man in der Auftragsverwaltung getrost von den selbstgesetzten Maßstäben ausgehen. Tenor und Tendenz der vornehmlich informellen Absprachen sind einem Diskussionspapier der Kultusdirektion zu entnehmen ${ }^{99}$. Ihnen lagen ähnliche Vorstellungen zugrunde, wie sie auch die deutsche Verwaltung während des ,Stuttgarter Interims“ formuliert hatte. Zur Entlassung kommen sollten neben den ,alten Kämpfern", die der Partei oder ihren Gliederungen schon vor 1933 angehört hatten, politische Leiter der NSDAP vom Ortsgruppenleiter aufwärts, hauptamtliche Funktionäre der Gliederungen sowie nicht näher definierte „Aktivisten und Denunzianten“. Sollten sich aus dem Grundsatz, daß kein Pg in leitender Stellung gehalten werden sollte, „zu große Schwierigkeiten“ ergeben, so konnte der beanstandete Beamte solange im Amt bleiben, bis sich eine "geeignete Lösung“ gefunden hatte. Zurückstufungen im Besoldungsdienstalter sollten ziemlich schematisch nach dem Beitrittsdatum zur NSDAP vorgenommen werden. Versetzungen sollten, außer bei der Polizei-Säuberung, die Ausnahme bleiben. In den Ruhestand versetzen wollte man Beamte, die das Pensionsalter ohnehin erreicht hatten. Insgesamt blieben solche Vorstellungen, die in der Auftragsverwaltung in großer Eigenständigkeit formuliert werden konnten, weit hinter dem Wortlaut der bisherigen französischen Direktiven zur Entnazifizierung zurück; nicht zu reden von der Entnazifizierungspolitik in der US-Zone. Das Staatssekretariat - es zeigte sich noch deutlicher in den folgenden Wochen - genoß in der Entnazifizierung einen Freiraum wie in keinem anderen Bereich der französisch kontrollierten Besatzungsverwaltung.

Die Prüfungen in den Kommissionen liefen gerade eine Woche, als man feststellen mußte, daß der Arbeits- und Zeitaufwand des Verfahrens stark unterschätzt worden war. In aller Eile wurde der Kreis der Kommissionsmitglieder erweitert. Dabei war die Innendirektion gezwungen, fast ausschließlich auf beiden Arbeiterparteien Nahestehende zurückzugreifen ${ }^{100}$.

Zwar trug diese Maßnahme und die Vereinfachung des Verfahrens ${ }^{101}$ zu einem zügigeren Fortgang der Säuberung bei, aber trotzdem rechneten die Fachleute noch im April damit, daß allein die Überprüfung der Innen- und Kommunalverwaltung noch

stehen nur eine verhältnismäßig geringe Anzahl geeigneter und politisch unbelasteter Personen zur Verfügung.“ Schreiben an einen Rechtsanwalt v. 9. 3. 1946; SIG, Wü 13/239.

99 „Richtlinien und Gesichtspunkte zur pol. Überprüfung der Beamtenschaft"; LdI an Direktorialamt v. 14. 2. 1946; SIG, Wü 13/154.

100 Von den zehn neu Vorgeschlagenen bezeichnete Roßmann gegenüber der Milreg acht als Linkssozialisten, SPD-Anhänger oder Kommunisten; Schreiben an die Milreg v. 27. 2. 1946; SIG, Wü 13/ 154.

101 Mitte März 1946 richtete G. H. Müller sogenannte „Unterkommissionen“ ein, die sich aus zwei ständigen Mitgliedern und einem Beamtenvertreter zusammensetzten. Bei Einstimmigkeit galt ein Fall als im Namen des Plenums entschieden; Schreiben an die Säuberungskommissionen v. 14. 3. 1946; SIG, Wü 13/154. 
„mehrere Monate“ in Anspruch nehmen werde ${ }^{102}$. Und eine Woche bevor der Vorsitzende der Säuberungskommission für die Innen- und Finanzverwaltung dem Fortgang der politischen Säuberung diese düstere Prognose stellte, hatte Carlo Schmid im Direktorium schon die Forderung erhoben, den offenkundigen Unzulänglichkeiten des Säuberungsverfahrens durch eine umfassende Rechtsanordnung ein Ende zu bereiten ${ }^{103}$. Diese ins Auge gefaßte Neuorientierung der Entnazifizierung führte zu einer Stagnation bei der politischen Überprüfung des öffentlichen Dienstes; jetzt bestand kaum noch Bereitschaft, die Säuberung nach einem Verfahren fortzuführen, das allenthalben als überholt galt. Lediglich die UUberprüfung der Polizeibeamten, als einziger Beamtengruppe, wurde noch nach dem alten Modus zu Ende geführt.

Die Militärregierung hatte wiederholt auf energische und rasche Säuberung der Polizei gedrungen. Da diese ihrer Auffassung nach „,das wirksamste Instrument zur Abschaffung der persönlichen Freiheit gewesen“" war, müsse sie radikal gesäubert werden. Die LdI sollte der Säuberungskommission einschärfen, auch in den leichten Fällen wenigstens Versetzungen vorzunehmen ${ }^{104}$. Nach den Ausführungen Roßmanns auf der Landrätetagung in Ravensburg deckte sich das Bestreben, einen scharfen Maßstab anzulegen, ,durchaus mit den bei der Landesverwaltung ohnehin bestehenden Absichten." Die Bewerber für den Polizeidienst durften zu keinem Zeitpunkt der NSDAP oder einer ihrer Gliederungen angehört haben. Doch dort, wo dieser Grundsatz die Funktionsfähigkeit der Verwaltung einzuschränken drohte, gelangte der Elan an seine Grenzen. Der Landesdirektor meinte nämlich weiter: „Eine Ausnahmeregelung wird freilich für Polizeioffiziere (!) getroffen werden müssen, um die Kontinuität der polizeilichen Arbeit zu gewährleisten" ${ }^{105}$. Am 15. März 1946 konnte Carlo Schmid der Militärregierung melden, daß in allen Kreisen sämtliche Angehörige der Polizei überprüft seien ${ }^{106}$. Von den Sanktionen waren praktisch nur Beamte betroffen, die der NSDAP angehört hatten ${ }^{107}$. Diese Reduktion auf eine reine Pg-Säuberung zeigt, daß die Bestimmungen der Richtlinien vom Herbst 1945, die in recht dehnbaren Formulierungen auch andere Merkmale politischer Belastung als nur die Mitgliedschaft in NS-Organisationen vorsahen, in der Praxis kaum Bedeutung gewannen. Die Aufforderungen des Direktoriums an die Kommission, „Polizei und Gendarmen unter Anlegung besonders strenger Maßstäbe “ ${ }^{108}$ zu prüfen, wirkte sich besonders auf die

102 Mitteilung der Säuberungskommission für Inneres und Finanzen an Landesdirektor Roßmann v. 6. 4. 1946; SIG, Wü 42/I, acc. 15/1974, 134.

103 DirS 26.3. 1946.

${ }^{104}$ Schreiben der Milreg an die LdI v. 19. 2. 1946; SIG, Wü 13/153. Aktenvermerk über Anruf von Hauptmann Vigouroux bei G. H. Müller v. 18. 2. 1946; SIG, Wü 13/154.

105 5. Lrtg in Ravensburg am 2.3.1946, S. 35.

106 Schreiben an die Milreg; SIG, Wü 13/154.

107 Von den ehemaligen Pg's unter den Beamten wurden 97,4 Prozent mit Sanktionen belegt; von den Nicht-Parteigenossen dagegen nur 1,8 Prozent. Die folgenden Angaben sind auf einer Basis von 865 Fällen berechnet und stammen aus dem Schlußbericht über die Polizeisäuberung der Säuberungskommission für Inneres und Finanzen an Landesdirektor Roßmann v. 20. 3. 1946; SIG, Wü 13/154. Insgesamt waren 1390 Beamte überprüft worden. 525 waren allerdings erst nach dem 1.4. 1945 in Dienst gestellt worden; 9. Lrtg in Saulgau am 6. 7.1945, S. 50.

108 DirS 22. 2. 1946. 
Versetzungs- und Gehaltskürzungsmaßnahmen aus ${ }^{109}$. Auch die Entlassenen-Quote lag wegen der wiederholten Ermahnungen zu scharfem Vorgehen leicht über dem Durchschnitt der KRUA-Vorschläge ${ }^{110}$. Ansonsten zeigte die zweite Instanz im Säuberungsverfahren, besonders bei den Entlassungsmaßnahmen, eine deutliche Tendenz zur Abschwächung der KRUA-Empfehlungen. Das ergibt sich aus einem Teilergebnis der Justizkommission. Bei den Entscheidungen auf Dienstentlassung, der tiefgreifendsten Maßnahme in der politischen Säuberung, lag die Kommission zum Teil erheblich niedriger als die Masse der KRUAS, von denen Vergleichszahlen vorliegen ${ }^{111}$. Daß man die Entscheidungen der Kommissionen nicht auch mit dem Vollzug dieser Maßnahmen gleichsetzen darf, zeigt eine Notiz der Justizdirektion, wonach dort bis zum 31. Mai 1946 im Zuge der politischen Säuberung noch immer „Entlassungen und Versetzungen nicht ausgesprochen" worden waren ${ }^{112}$.

Am Charakter der politischen Säuberung in Südwürttemberg änderten auch die Bestimmungen der Kontrollratsdirektive Nr. 24 nichts, denn die Franzosen führten sie, wie Schmid dem Stuttgarter Kabinett berichtete, ,auf ihre Weise“, das hieß: gar nicht durch. „Sie werden wohl nachher erklären“, prophezeite Schmid, „daß sie mit der Ausfïhrung schon fertig seien "113. Wohl hatte die Militärregierung im November, als die Direktive im Kontrollrat beraten wurde, dem Staatssekretariat die alliierte Vereinbarung in einem Erlaß vorgeschrieben, wonach kein Beamter, der in einer Zone entlassen worden war, in einer anderen wieder eingestellt werden durfte ${ }^{114}$. Doch eine ganze Reihe von Beamten, auf die diese Bestimmungen eigentlich zutrafen, hatten ihren Dienst in Südwürttemberg schon vor diesem Erlaß angetreten. Die Militärregierung legte dann auf eine rückwirkende Anwendung ihrer Anweisung keinen Wert, auch nicht nach Verabschiedung der Kontrollrats-Direktive. So fand das Beschäftigungsverbot für Beamte aus anderen Zonen im französisch besetzten Gebiet keine Anwendung. Das blieb so, bis der parlamentarische Untersuchungsausschuß der französischen Nationalversammlung im Februar die Verhältnisse in der Zone unter die Lupe nahm. Ohne Mühe konnten die Parlamentarier dem Gouvernement Militaire nun nachweisen, worüber sich in Frankreich seit langem die Gemüter erhitzt hatten: nämlich die Mißachtung der gemeinsamen alliierten Beschlüsse über die Entnazifizierung in Deutschland. Dabei spielte es im Augenblick keine Rolle, welche Maßstäbe zu den Entlassungen in der amerikanischen Zone geführt hatten und daß viele der mit Sanktionen Belegten, die nun im französischen Besatzungsgebiet Dienst taten, alles

109 KRUA-Durchschnitt war 13,5 Prozent gewesen.

110 Knapp 10 Prozent gegenüber 7,5 Prozent aller Fälle.

111 Die Entlassungsquote bei den Entscheidungen der Justizkommission betrug 4,3 Prozent (berechnet auf einer Basis von 715 Fällen); Statistik zur Säuberung des deutschen Personals der LdJ vom 29. 3. 1946; StAL, Akten der LdJ, 2014-21.

112 Schreiben der LdJ an das Direktorialamt v. 31.5. 1946 (StAL, Akten der LdJ, 2014-28) auf dessen Erlaß v. 18.3.1946 (SIG, Wü 140/2800).

113 Kabinettssitzung des StaMi in Stuttgart am 19.2. 1946; HStASt EA 1/20 X 8.

114 Diese Bestimmung erschien in der Kontrollrats-Direktive Nr. 24 unter Punkt 10., 99. Erlaß der Milreg an das Staatssekretariat v. 5.11. 1945; SIG, Wü 120/P 1016 A/25/590. S.a. den Umlauf in der LdJ v. 10. 12. 1945; JuMinSt, Akten der LdJ von Württemberg-Hohenzollern, $9133 \mathrm{~h}$. 
andere als eingefleischte Nazis waren. Die Militärregierung geriet wieder einmal in Zugzwang. Die überstürzte Reaktion der französischen Militärbehörden traf das Staatssekretariat wie ein Blitz aus heiterem Himmel: Bis zum 15. März, hieß es nun plötzlich, müßten alle Beamten der Auftragsverwaltung, die irgendwann „Gegenstand von Säuberungsmaßnahmen seitens alliierter Behörden " gewesen waren, entlassen sein. Diese Maßnahmen sollten sich auf alle Personen erstrecken, die - wie es in Anlehnung an die Bestimmungen der Kontrollratsdirektive hieß - in Unternehmungen von ,allgemeinem Interesse“ beschäftigt waren. Wer sich nach dem Stichtag noch im Dienst befand, sollte ,automatisch Gegenstand einer Internierungsmaßnahme“ wer$\operatorname{den}^{115}$ ! Für Ausnahmeregelungen mußten begründete Anträge gestellt werden. Allein in den Tübinger Zentralbehörden fielen 42 Beamte unter diese Bestimmungen. Manchem Betroffenen mag bewußt gewesen sein, wer ebenfalls zu den in der amerikanischen Zone Entlassenen zählte: Carlo Schmid selbst. Die Hinweise der Landesdirektoren, sie hätten bei der Einstellung ihrer Beamten doch die ,ausdrückliche Zustimmung" der Fachabteilungen der Militärregierung eingeholt, halfen zunächst nichts ${ }^{116}$. Einige Direktionen begannen in den untergeordneten Dienststellen tatsächlich mit der „vorsorglichen“ Entlassung. Die betroffenen Beamten in den Zentralbehörden blieben solange im Amt, bis die Militärregierung über die Ausnahmeanträge entschieden hatte, die vom Staatssekretariat für fast alle Betroffenen gestellt worden waren. Die Entscheidung der Militärregierung Ende April war für das Staatssekretariat ein neuerlicher Schock. Nur drei der 42 Beamten, die entlassen werden sollten, durften weiterbeschäftigt werden ${ }^{117}$. Den Hintergrund dieses ungewohnt drastischen Vorgehens ließ der Säuberungskommissar der Militärregierung, Hauptmann Vigouroux, gegenüber einem Beamten der Innendirektion durchblicken, als er andeutete, ,daß man Zahlenmaterial für höchste Stellen benötige" 118 . Doch sogleich, als es galt, auf der Durchführung der eigenen Anordnungen $z \mathfrak{u}$ bestehen, zeigte sich, daß die Militärregierung wieder einen ,coup de thêatre' gelandet hatte. Nach und nach traf jeder Verwaltungszweig mit dem Gouvernement Militaire sein gentlemen's agreement ${ }^{119}$. Der ,modus vivendi“", wie G. H. Müller es nannte ${ }^{120}$, war schnell gefunden. Mit der Auflage, die deutschen Säuberungsorgane sollten sich demnächst mit ihnen befassen, blieben die Beamten, die nach der Anordnung vom 26. April eigentlich „sofort zu entlassen“ gewesen waren, weiterhin im Amt. Als der Untersuchungsbericht der französischen Kommission in Frankreich bekannt wurde, sah sich das Gouvernement Militaire

115 Erlaß der Milreg an das Staatssekretariat v. 20. 2. 1946; SIG, Wü 42 P, acc. 10/1970, A 13150.

116 Schreiben der Kultusdirektion an die Milreg v. 26.2.1946; SIG, Wü 80, acc. 10/1974, 56.

117 Schreiben des Gouverneurs an das Staatssekretariat v. 26. 4. 1946; SIG, Wü 120/P 1016 A/26/ 591.

118 Aktenvermerk der LdI v. 15. 3. 1946; SIG, Wü 42 P, acc. 10/1970, A 13045.

119 Dazu die Aktenvermerke von Gebhard Müller v. 16. 5. und 17. 5. 1946; StAL, Akten der LdJ, 2014 - 2. Brief der LdF an den Staatskommissar für die politische Säuberung v. 24. 5. 1946; SIG, Wü 120/P 1016 A/29/594. Aktenvermerk der Innenverwaltung v. 29.4.1946; SIG, Wü 42 P, acc. 10/1970, A 13150/24.

120 Aktennotiz von G. H. Müller v. 19. 7. 1946; SIG, Wü 42 P, acc. 10/1970, A 13150/46. 
gezwungen, erneut zu der schon erprobten Taktik der Scheinradikalität und der Verschleierung Zuflucht zu nehmen. Dabei ging es diesmal nicht ohne eine regelrechte Erpressung der neu zugelassenen politischen Parteien ab.

\section{Die Erpressung der politischen Parteien durch die Militärregierung}

Zwischen dem Eklat um die Entnazifizierungspolitik der Militärregierung und der Lizenzierung der politischen Parteien in Südwürttemberg am 18. März 1946 besteht ein direkter Zusammenhang. In einem ausgeklügelten Manöver versuchte die Besatzungsmacht, mit diesem Schritt sowohl die Angriffe auf den desolaten Zustand der politischen Säuberung abzuwehren, als auch der Forderung des Untersuchungsausschusses Genüge zu tun, auch die französische Zone müsse nun endlich Anschluß an die parteipolitische Entwicklung der anderen Zonen Anschluß finden. Die Zulassung der Parteien ermöglichte es nämlich dem Gouvernement Militaire, noch am gleichen Tage die Bildung einer Kommission aus Vertretern der drei Parteien (CDU, SPD, KPD) ,als oberste Instanz in allen Säuberungssachen “ anzuordnen ${ }^{121}$. Welche Motive veranlaßten die Militärregierung zu diesem spektakulären Schritt? Wie ist es zu erklären, daß die eben zugelassenen Parteien, die noch keine nennenswerte Resonanz in der parteipolitisch abstinenten Bevölkerung hatten ${ }^{122}$, und die ansonsten unter den gegebenen Bedingungen noch keinerlei Einfluß im Sinne einer demokratischen Willensbildung auf die Auftragsverwaltung geltend machen konnten, nun auf einmal in einer so hochpolitischen Angelegenheit das letzte Wort haben sollten? Die nähere Betrachtung ergibt, daß die Militärregierung, von grundsätzlichen Erwägungen demokratischer Prinzipien - wie der Gouverneur glauben machen wollte - weit entfernt, dieses sogenannte Kontrollorgan aus rein taktischen Überlegungen ins Leben gerufen hatte. Denn wieder einmal war sie unter Druck von außen geraten. In diesem Falle ging er wieder von der Parlamentarischen Untersuchungskommission der Französischen Nationalversammlung aus.

Bei ihrer Untersuchung des Säuberungsverfahrens in der französischen Zone war die Enquête-Kommission einer wichtigen Ursache für das mangelhafte Resultat der Entnazifizierung auf die Spur gekommen. Sie sah die Hauptursache für die unhaltbar unterschiedliche Arbeitsweise der Säuberungsorgane in deren personeller Struktur. Viele Ausschüsse, hieß es im Schlußbericht des Untersuchungsausschusses, seien hinsichtlich der politischen Haltung ihrer Mitglieder völlig einseitig zusammengesetzt.

121 GouvS 18.3.1946.

122 Als Beispiel eine typische Aussage zur Haltung der Bevölkerung gegenüber den Parteien aus dem „Bulletin d'Activité“ des C. C. F. A. vom März 1946: „Leurs Chefs [der Parteien] essayent, en général, d'utiliser la liberté politique qui leur est accordée pour rassembler autour d'eux les énergies allemandes. Mais ces efforts se heurtent à la torpeur et à l'apathie de la grande masse du public allemand. L'ensemble de la population ne s'interesse ni aux réunions politiques, ni à la propagande entreprise en faveurs des diverses programmes ...: elle est beaucoup plus préoccupée par son ravitaillement présent et sa situation materielle à venir." IfZ-Archiv. 
Die Verantwortung hierfür lastete er zu Recht der Militärregierung an; die Überwachung der deutschen Personalvorschläge sei zu nachlässig gehandhabt worden. Denn wenn die Militärbehörden von Anfang an nur Ausschüsse in der von ihr ausdrücklich vorgeschriebenen Zusammensetzung genehmigt hätte, so folgerten die Abgeordneten, dann hätten sich diese schweren Mängel und dieses insgesamt unbrauchbare Resultat vermeiden lassen. Dieses Versäumnis der Militärregierung erkläre, „,que la dénazification définitive se fasse lentement et mal" 123 . Auch auf die schwammigen und schlecht ausgearbeiteten materiellen Vorschriften für die Säuberung dürften die Mitglieder der Kommission während ihres Aufenthaltes in der Zone gegenüber den Verantwortlichen der Militärregierung zu sprechen gekommen sein.

In Reaktion auf die Vorwürfe des Untersuchungsausschusses, der in eben diesen Tagen der Französischen Nationalversammlung seinen detaillierten Schlußbericht vorlegte ${ }^{124}$, griff das Gouvernement Militaire nun auf seine bewährte Taktik zurück. Der ,politische Säuberungsrat" sollte nach dem Erlaß des Gouverneurs ${ }^{125}$ als oberstes Kontrollorgan der Entnazifizierung für die Vereinheitlichung der Sanktionen sorgen und die ausgewogene Zusammensetzung der Säuberungsorgane sicherstellen. Mit diesem Auftrag war der Parteienrat genau auf die beiden Problemkreise angesetzt, zwischen denen auch die Enquête-Kommission einen direkten Zusammenhang hergestellt hatte. Daneben trat der Parteienrat, aufgrund seiner Akkreditierung beim Gouverneur, als ein von der Auftragsverwaltung unabhängiges, quasi-parlamentarisches Kontrollgremium in Erscheinung. Doch alle Aufgaben des politischen Säuberungsrates mußten unter den gegebenen Verhältnissen Fiktion bleiben. Das demokratische Kontrollprinzip, das der Gouverneur in der ersten Besprechung mit den drei Parteivertretern eigens herausstellte, konnte unter den Bedingungen der frühen Besatzungszeit nicht verwirklicht werden. Denn es bestand kein Interessengegensatz zwischen der Auftragsverwaltung und den Parteien. Vielmehr waren die führenden Repräsentanten der Parteien, von der KPD abgesehen, selbst an hervorragender Stelle der Auftragsverwaltung tätig. Die Beteiligung der KPD fiel nicht ins Gewicht, da für eine Beschlußfassung im Parteienrat keine Einstimmigkeit vorgeschrieben war. Daß der Beirat von sich aus eine gemeinsame politische Linie gegen die Säuberungsgremien verfolgen würde, in denen ebenfalls die Vertreter von SPD und CDU, bzw. die Beamten der Auftragsverwaltung die Mehrheit hatten, war aufgrund der politischen Verhältnisse im Staatssekretariat ausgeschlossen. Der südwürttembergischen Landesverwaltung hatte als einziger deutscher Auftragsverwaltung von Anfang an kein Kommunist in führender Stellung angehört. Das hatte, nach dem Zeugnis Theodor Eschenburgs, damals selbst führender Beamter im Staatssekretariat, „Schmid zu verhindern“ gewußt ${ }^{126}$. Deswegen änderte es auch an der Qualität der Entscheidungen der Säuberungskommissio-

123 Assemblée, Documents, Annexe Nr. 924.

124 Assemblée, Débats, 8. 4. 1946, S. 1593.

125 Schreiben von Gouverneur Widmer an das Staatssekretariat v. 4. 4. 1946; SIG, Wü 13/154. S. a. das Schreiben der französischen Militärregierung Baden an die Badische Landesverwaltung v. 18. 3. 1946 zum gleichen Gegenstand; StAF, Bad. Staatskanzlei, 8004.

126 Eschenburg, Anfänge, S. 269. 
nen nichts, daß nun einige Kommunisten neu aufgenommen wurden ${ }^{127}$. Eine Umbildung der KRUAS von Grund auf kam für die Militärregierung ohnehin nicht in Frage, denn dies wäre dem offenen Eingeständnis eines kompletten Fehlschlages der Säuberungsbemühungen des letzten halben Jahres gleichgekommen.

Möglicherweise hatte es unter den Kritikern der Militärregierung aufmerksame politische Beobachter gegeben, die hinter den weitreichenden Befugnissen des „obersten Kontrollorganes" dessen faktische Einflußlosigkeit erkannt hatten. Doch selbst sie hätten jetzt ihre Zweifel an einem wirklichen Umschwung der Entnazifizierung in der französischen Zone begraben müssen. Denn der politische Säuberungsrat verabschiedete nach seiner ersten Sitzung mit dem Gouverneur zur allgemeinen Überraschung eine Resolution zur Entnazifizierung in Südwürttemberg, die in ihrer Radikalität alles in den Schatten stellte, was man bisher von deutscher Seite hierzu jemals vernommen hatte. Nach dem Beschluß des Beirates mußten 50 Prozent der Beamten, die der NSDAP angehört hatten, aus dem öffentlichen Dienst entlassen werden. Aus Wirtschaftsunternehmungen mit mehr als hundert Beschäftigten mußten „75 Prozent der am meisten belasteten Betriebsführer" gehen; für Betriebe mit weniger Beschäftigten veranschlagte der Beirat die Entlassungsquote auf 50 Prozent. Ihr Vermögen sollte enteignet und der Allgemeinheit zur Verfügung gestellt werden ${ }^{128}$. Die Durchführung dieses Beschlusses, der einer politischen Sensation gleichkam, hätte zu einem Vielfachen der Entlassungsquoten geführt, die selbst die schärfsten KRUAS gefordert hatten. Jedoch: Dieser spektakuläre „Beschluß“ entsprang keineswegs einer freien Meinungsbildung der Parteien, sondern einem Diktat des Gouverneurs! Er mißbrauchte die eben zugelassenen Organe demokratischer Willensbildung rücksichtslos zur Stabilisierung der angeschlagenen Position des Gouvernement Militaire. Deutlich wie selten enthüllte sich in diesem zynischen Manöver die opportunistische Gleichgültigkeit, mit der die Militärregierung der Demokratisierung des politischen Lebens in ihrem Einflußbereich gegenüberstand. Diese Machenschaften standen in einem eklatanten Widerspruch zu öffentlichen Bekenntnissen und sogar zu der internen Darstellung im „Bulletin d'activité“, nach der die Zulassung der politischen Parteien der deutschen Bevölkerung „des moyens efficaces d'exprimer leurs opinions et leurs voeux “129 an die Hand gegeben hätten.

Das nachfolgende Dokument entstammt den Aufzeichnungen, die der CDU-Vertreter im Säuberungsrat, Ministerialrat Dr. Gekle, von dieser Besprechung mit dem Gouverneur anfertigte ${ }^{130}$ :

127 Zur Umbesetzung vergl. z. B. das Schreiben der LdJ an die Justizbehörden v. 30. 4. 1946; SIG, Wü 13/158.

${ }^{128}$ Beschlüsse des Politischen Kontrollkomitees für die Säuberung vom 6. 4. 1946; SIG, Wü 80, acc. 10/1974, 56 und StAL, Akten der LdJ, 2014-31/3.

129 Bulletin d'activité, Januar 1946, S. 4. IfZ-Archiv.

130 Gebhard Müller bemerkte in einer Information an den Verfasser, daß es seinerzeit sehr riskant gewesen sei, Aktenvermerke dieser Art anzulegen, „da man nie sicher war, ob sie nicht in die Hände der Sûretéc" gelangten. Unter diesem Gesichtspunkt seien die folgenden Aufzeichnungen „sehr weit" gegangen. 
„1. Am Samstag, den 6. April, vormittags $11 \mathrm{Uhr}$ wurde ich als Vertreter der CDU zur Französischen Militärregierung hier (Zimmer 34) zu einer Besprechung bestellt. Wenige Tage vorher wurde mir vom Direktorialamt des Staatssekretariats hier mitgeteilt, es handle sich um eine Besprechung über Fragen der Entnazifizierung. In den großen Sitzungssaal des Landgerichts geführt, fand ich dort einen Herrn Acker, Vertreter der Kommunistischen Partei, und Stadtrat Bartels, Vertreter der SPD, bereits vor. Die Vertreter der 3 politischen Parteien wurden vom Franz. GeneralGouverneur, General Widmer, und einer Anzahl anderer Offiziere vorgestellt und durch Handschlag begrüßt.

2. General Widmer machte in französischer Sprache, die von einem Dolmetscher alsbald ins Deutsche übersetzt wurden, Ausführungen über den Zweck unseres Kommens:

Einschaltung der politischen Parteien als eine Art Kontrollorgan bei der im Gange befindlichen Entnazifizierung, ein Organ, demgegenüber Anregungen, Wünsche und Beschwerden der 3 Parteien vorgebracht werden können. Denn bei der Entnazifizierung soll auch dem demokratischen Prinzip Rechnung getragen werden. [...]

II.

Hierauf machte der General-Gouverneur folgende schwerwiegende Ausführungen:

Die Französische Militärregierung sei der Auffassung, daß die zuständigen Säuberungskommissionen folgende Entlassungen der meist belasteten Nazianhänger gleichgültig von wann ab ihr Eintritt in die Partei erfolgt sei - vorzunehmen hätten: [Es folgen die oben genannten Prozentsätze und Bestimmungen.]

III.

Auf meine weiteren Einwendungen, daß es nicht tragbar erscheine, einen so hohen Prozentsatz von Beamten und Angestellten aus öffentlichen Verwaltungen zu entlassen, da es insbesondere an entsprechendem Nachwuchs fehle, wurde eingewandt, dies müsse auch in Württemberg möglich sein wie anderwärts, entweder seien wir bereit zu entnazifizieren oder nicht. Meiner weiteren Bitte, in das Protokoll die Tatsache aufzunehmen, daß die Höhe des Prozentsatzes der vorzunehmenden Entlassungen auf Vorschlag des Herrn General-Gouverneurs erfolgt sei, wurde nicht stattgegeben mit der Begründung des Dolmetschers: ,Warum wollen Sie keine Verantwortung hierfür auf sich nehmen? Entweder wollen Sie entnazifizieren oder nicht'.

Auf die Einwendungen des Vertreters der SPD, ob man schematisch diesen Prozentsatz der Entlassungen einhalten müsse, erklärte der Gouverneur durch den Dolmetscher: Ja, dieser Prozentsatz sei der mindeste Satz, der bei der Entlassung zur Anwendung kommen soll, man könne ruhig darüber hinausgehen.

[...]

Nach Lage der Sache hatte ich den Eindruck, daß weitere Einwendungen erfolglos gewesen sein würden.

$[\ldots]$ 
Wir wurden gebeten, diese Niederschrift zu unterschreiben. Hierauf wurden wir vom Herrn General-Gouverneur entlassen mit der Erklärung: Auf Wiedersehn meine Herren am nächsten Freitag $15.30 \mathrm{Uhr}^{\text {“"131. }}$

Noch am selben Tage unterrichtete der Gouverneur das Staatssekretariat offiziell von der Resolution des Parteienrates. „Diese Vorschläge“, so führte er aus, „erscheinen mir vollkommen gerechtfertigt." Er fügte noch hinzu, „daß es sich nach den Gedankengängen des Komitees“ bei den Entlassungsquoten um „Mindestziffern“ handele, über die im einzelnen noch hinausgegangen werden könne ${ }^{132}$. Er ordnete an, diese Richtlinien sofort den Säuberungskommissionen für die Verwaltung bekannt zu machen. Da es im Staatssekretariat natürlich kein Geheimnis geblieben war, daß der Gouverneur selbst dem Parteienrat diese „Vorschläge“ und „Gedankengänge“ zur politischen Säuberung aufgezwungen hatte, und da man selbstverständlich auch in der Militärregierung davon ausgehen mußte, daß dem Staatssekretariat die Umstände, die zu dem Parteienbeschluß geführt hatten, sogleich zur Kenntnis gelangen würden, ist es offensichtlich, daß dieses Schreiben vor Dritten, die diese Hintergründe nicht kannten, Potemkinsche Dörfer errichten sollte. Als eigentliche Adressate des Schreibens hatte die Militärregierung die kritischen Parlamentarier der Französischen Nationalversammlung im Auge, die schon während ihres Aufenthaltes in der Besatzungszone das Recht zur Einsichtnahme in die Akten der Besatzungsbehörden gehabt hatten. Ihnen konnten nun jederzeit Beweise vorgelegt werden, daß man es in der Besatzungszone nicht mehr am nötigen Nachdruck bei der politischen Säuberung fehlen ließ.

In Wahrheit - so zeigte sich alsbald - war die Militärregierung aber wieder nicht an der Einhaltung der neuen Bestimmungen durch die deutschen Säuberungsorgane interessiert. Sie wich auch jetzt nicht von ihrer Politik der Scheinradikalität ab, die sie seit dem Herbst in der politischen Säuberung Südwürttembergs verfolgt hatte: Wenige Tage nach dem erwähnten Schreiben an das Staatssekretariat, in dem sie die radikalen „Beschlüsse“ des Parteienrates bekräftigt hatte, nahm die Militärregierung diese Bestimmungen intern und mündlich wieder zurück. Dem Staatskommissar für die politische Säuberung, der in diesen Tagen ernannt worden war, wurde nämlich signalisiert, daß er ,an die am 6. April festgelegten Entlassungsquoten nicht gebunden sei““133.

131 Vertraulicher Aktenvermerk von Ministerialrat Dr. Gekle (CDU) v. 6. 4. 1946; StAL, Akten der LdJ, 2014-31/3. Hervorhebung im Original. Die Führung der CDU von Südwürttemberg kam bei der Beratung dieses Beschlusses zu dem Ergebnis, „daß Dr. Gekle in dieser schwerwiegenden Frage die Unterschrift ohne Zustimmung der Vorstandschaft nicht hätte unterzeichnen dürfen. In derart schwerwiegenden Angelegenheiten könne ein einzelner Vertreter nicht für die ganze Partei eine bindende Erklärung abgeben." StAL, Akten der LdJ, 2014-31/3a.

132 Schreiben von Gouverneur Widmer an das Staatssekretariat v. 6. 4. 1946; SIG, Wü 80, acc. 10/ $1974,56$.

133 Tätigkeitsschlußbericht des Staatskommissars für die politische Säuberung v. 18. 5. 1948; SIG, Wü 2, I, 610/14/6. Der eigentliche Charakter des Manövers der Milreg, die „nicht ganz frei von schematischem Denken“ gewesen sei, blieb auch Fürstenau verborgen. Das Abrücken des Gouvernement Militaire von ihren Entlassungsquoten stellt sich ihm als ein Verhandlungserfolg Künzels dar. Wegen dessen unnachgiebiger Haltung in den Verhandlungen mit den französischen Behörden hätte der Gouverneur dann schließlich versprochen, „die Prozentforderungen fallenzulassen" (S. 140). 
Infolge der Einsetzung des Parteienrates - so einflußlos dieses Gremium künftighin auch blieb - konnte das Kommissionsverfahren nicht mehr in seiner bisherigen Form weitergeführt werden. Solange nicht die praktische Umsetzung der neuen Bestimmungen geklärt war, hatte eine „Weiterarbeit der Säuberungskommissionen keinen

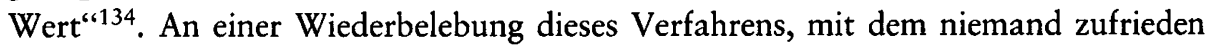
war, bestand kein Interesse. Stattdessen ging man nun im Staatssekretariat daran, gemäß einer Forderung Carlo Schmids das gesamte System der Entnazifizierung in Südwürttemberg durch „eine umfassende Rechtsanordnung““135 neu zu regeln.

\section{Anfänge der politischen Ưberprüfung der Wirtschaft}

In den ersten Wochen nach dem Erhalt der Richtlinien für die politische Säuberung der Beamtenschaft fragte man sich im Tübinger Staatssekretariat zunächst weniger, nach welchen Kriterien nun die Entnazifizierung der freien Wirtschaft zu erfolgen habe, sondern ob die französische Militärregierung überhaupt deren politische Überprüfung anordnen werde ${ }^{136}$. Auch innerhalb des Gouvernement Militaire war die Meinungsbildung hierzu im Oktober offenbar noch nicht abgeschlossen. Es sei „fraglich“, so wurde dem Direktorium mitgeteilt, „ob im französisch besetzten Gebiet eine besondere Säuberungsaktion für die Wirtschaft eingeleitet wird“137.

Wenige Wochen später stand dann aber doch fest, daß auch die freie Wirtschaft einem Säuberungsverfahren unterzogen werden sollte. In einer Vorbesprechung betonte Kabinettschef de Mangoux, die Militärregierung wolle sich nicht in dieses Verfahren einmischen, es liege ausschließlich in den Händen des Staatssekretariats. Die von den Landesdirektionen vorgeschlagenen Maßnahmen sollten dem Gouverneur nur vorgelegt werden, „um zu vermeiden, daß allzu scharfe Kommissionen das Wirtschaftsleben lahmlegen." Denn das Hauptziel der Wirtschaftssäuberung sei es, die Unsicherheit bei den Geschäftsleuten zu beseitigen, die ihre „wirtschaftliche Aktivität"lähme. Auch dort, wo jemand politisch belastet sei, solle er weiter in seiner Stellung belassen werden, ,wenn er bisher durch die Tat bewiesen hat, daß er zu loyaler Zusammenarbeit mit der jetzigen Regierung bereit" sei ${ }^{138}$.

Die Richtlinien der Militärregierung zur politischen Säuberung der freien Wirtschaft ergingen am 3. Dezember $1945^{139}$. Von der Säuberungsaktion waren die, ,industriellen, handwerklichen, Handels-, Landwirtschafts- und Finanzbetriebe“ betroffen. Zur Uberprüfung kommen sollten zum einen „die Unternehmer, die Mitglieder des Auf-

134 Schreiben des Vorsitzenden der Säuberungskommission für die Innen- und Finanzverwaltung an das Staatssekretariat v. 11. 4. 1946; SIG, Wü 13/57.

135 DirS 26.3. 1946.

136 DirS 23. 10. 1945. S.a. die Anfragen des Landrates von Rottweil an die LdI v. 14. 11. 1945 (SIG, Wü 140/76/6000) und der „Demokratischen Vereinigung“, Tübingen, v. 8.11. 1945 (ebd.).

137 Mitteilung der Milreg an das Staatssekretariat; DirS 26. 10.1945.

138 Besprechung der Landesdirektoren mit Oberst de Mangoux; GouvS 20.11. 1945. In der DirS 27. 11. 1945 berichtete der Landesdirektor für Wirtschaft, dem Staatssekretariat werde in der Wirtschaftssäuberung von der Milreg ,völlig freie Hand“ gelassen werden.

139 Schreiben des Gouverneurs an den Vorsitzenden des Staatssekretariats; SIG, Wü 15/514. 
sichtsrates und der Direktion, die leitenden Ingenieure und Abteilungsleiter" aller Betriebe, die mehr als 20 Arbeitnehmer beschäftigten, bzw. über mehr als 500000 RM Stammkapital verfügten ${ }^{140}$. Das betraf in Südwürttemberg etwa 1500 Betriebe $^{141}$. Die Säuberungsrichtlinien erstreckten sich zum anderen, unabhängig von Kapital und Beschäftigtenzahl des Betriebes, auf alle Personen des wirtschaftlichen Lebens, für die aufgrund ihrer politischen Vergangenheit „das endgültige Verbot jeglicher leitenden oder Schlüsselstellung in Frage käme." Hier war der deutschen Seite also ein beträchtlicher Spielraum gelassen.

Wie bei der Verwaltungssäuberung war auch bei der politischen Uberprüfung der Wirtschaft ein zweistufiger Säuberungsapparat vorgesehen. Die Untersuchungsausschüsse in den Kreisen setzten sich aus fünf Mitgliedern zusammen, wobei die Kapitaleigner und die leitenden Angestellten ein Übergewicht besaßen ${ }^{142}$. Dieser Ausschuß hatte die Angaben der Betroffenen in den Fragebogen zu prüfen und diese dann in die sogenannten a-Fälle, b-Fälle und c-Fälle einzuteilen. Die Akten der a-Fälle, auf die keinerlei Belastung im Sinne der Richtlinien zutraf, waren abzuschließen und der Landesdirektion der Wirtschaft zur Verwahrung zu übersenden. $\mathrm{Zu}$ den b-Fällen gehörten alle Personen, die das Kriterium einer Formalbelastung erfüllten, die in einer anhängenden Liste aufgeführt war. Diese ist mit jener ominösen Liste identisch, die auch den Richtlinien zur Verwaltungssäuberung beigelegen hatte. Die Akten dieser Fälle waren mit einem Sanktionsvorschlag zu versehen und an die zweite Instanz, die Säuberungskommissionen, weiterzuleiten. Die Akten der sogenannten c-Fälle hatten die Ausschüsse nur zusammenzustellen und ohne eigenen Vorschlag an die Kommissionen weiterzuleiten. $\mathrm{Zu}$ dieser Betroffenen-Gruppe gehörten alle Personen, „die sich“, wie es hieß, „Denunziationen bei den nationalsozialistischen Behörden haben zuschulden kommen lassen, oder Personen, deren Tätigkeit einen solchen Charakter hatte, daß die Beibehaltung in einer Stellung angetan wäre, die Wiederherstellung der demokratischen Freiheiten und Einrichtungen in Deutschland zu gefährden." Bis auf den letzten Zusatz fand sich diese Formel schon in ähnlicher Fassung in den Richtlinien zur Entnazifizierung der Verwaltung. Da die Formel nicht näher definiert war, eröffnete sich hier auch der zweiten Säuberungsinstanz ein weiter Ermessensspielraum. Zweierlei Sanktionen waren vorgesehen. Etwas unklar unterschied die Militärregierung zwischen „,beruflichen“ und ,persönlichen“ Zwangsmaßnahmen. Zum einen konnte ein ,z.eitweises oder endgültiges Verbot, irgendeine leitende Tätigkeit auszuüben“, verhängt werden. Zum anderen war Aufenthaltsverbot in einem bestimmten örtlichen Bereich vorgesehen sowie eine „teilweise oder vollständige Be-

140 Mit Rücksicht auf die besondere Struktur der südwürttembergischen Wirtschaft zog die Milreg die Beschäftigtengrenze bei 20 Arbeitern und Angestellten. In anderen Ländern ihrer Zone galten die Bestimmungen erst bei Betrieben mit mehr als 50 Angestellten. Schreiben von General Schwartz (Milreg Baden) an die Landeswirtschaftskammer Baden v. 28. 11.1945; StAF, Bad. Innenministerium, Nr. 83.

141 DirS 7. 12. 1945.

142 Die Ausschüsse setzten sich zusammen aus je einem Unternehmer oder leitenden Direktor, einem Vertreter der Abteilungsleiter, einem Vertreter des Meisterpersonals und je einem Arbeiter und Angestellten. 
schlagnahme des Vermögens oder Zwangsverwaltung dieses Vermögens.“ Die Ausarbeitung detaillierter Richtlinien war ganz der deutschen Verwaltung anheim gestellt. Das bedeutete, daß - ganz gleich wie sich die Säuberungskommissionen schließlich zusammensetzten - eine vollständige Kontrolle der Wirtschaftsdirektion über alle vermögensrechtlichen Maßnahmen gewährleistet war.

Die Säuberungskommissionen, die Entscheidungsinstanzen in diesem Verfahren, bestanden aus sieben Mitgliedern. Fünf aus den verschiedenen Gruppen der Betriebsangehörigen (wie bei den Kreisausschüssen), einem Beamten der Wirtschaftsverwaltung sowie einem ,aus den bekannten Gegnern des Nationalsozialismus oder den Opfern gewählten Vertreter.“ Der politische Aspekt der Entnazifizierung war durch diese Zusammensetzung gegenüber dem Verfahren gegen Verwaltungsangehörige natürlich deutlich reduziert. Aufgestellt wurde die Mitgliederliste der Kommissionen von der Wirtschaftsdirektion.

Ausdrücklich wurde darauf hingewiesen, daß „weder zwischen den Untersuchungsausschüssen und den lokalen Militärregierungen, noch zwischen den Säuberungskommissionen und der regionalen Militärregierung ein direkter Verkehr bestehen darf. Die Offiziere der Militärregierung haben in keiner Weise bei den Beschlüssen ... mitzuwirken". Damit hatte das Gouvernement Militaire in Tübingen den Einfluß derjenigen Kreisgouverneure ausgeschaltet, die, besonders in den ersten Monaten der Besetzung, sogenannte „wilde Säuberungen“ durch örtliche Antifa-Komitees unterstützt hatten. Diese Art von Säuberungsmaßnahmen müßten „selbstverständlich ... vom Prüfungsausschuß grundsätzlich nochmals geprüft" werden, meinte der Landesdirektor für Wirtschaft, Dr. Gustav Kilpper, auf einer Landrätetagung ${ }^{143}$. Auch der Gouverneur beanstandete gegenüber Carlo Schmid, daß an einigen Orten ,wilde Säuberungsausschüsse“ tätig seien. Die Säuberung könne „ausschließlich nur durch die amtlich bestellten Ausschüsse erfolgen" ${ }^{\text {"144 }}$. Die verschiedenen Antifa-Vereinigungen hielt Widmer für ,"überflüssig“" ${ }^{145}$. Die Säuberungsaktion sollte zwar nicht mehr „vor den Festen zum Jahresende“ beginnen, mußte aber unter allen Umständen bis Mitte März abgeschlossen sein, da die „Störung des Wirtschaftslebens auf ein Mindestmaß herabgesetzt" werden müsse.

Der Kontrast dieser Bestimmungen zu den Vorschriften über die Wirtschaftssäuberung, die im benachbarten Nordwürttemberg in Kraft waren, konnte nicht größer sein. Dort mußten nach dem amerikanischen Militärregierungsgesetz Nr. 8 alle ehemaligen Mitglieder der NSDAP oder deren Gliederungen bzw. angeschlossenen Verbänden aus dem Erwerbsleben ausscheiden, soweit sie in ihren Anstellungen mehr als nur ,gewöhnliche Arbeit" verrichteten ${ }^{146}$. Dieses Gesetz, von den amerikanischen

143 3. Lrtg in Reutlingen am 3. 1. 1946, S. 22.

144 GouvS 8. 1.1946.

145 GouvS 22. 1. 1946. Zu Struktur und Funktion der Antifa-Bewegung, vergl. Arbeiterinitative 1945 (dort auch umfassende Literaturhinweise) sowie Niethammer, Aktivität und Grenzen.

146 Military Government Germany, United States Zone, Law Nr. 8: Prohibition of Employment of Members of the Nazi. Party in Positions in Business other than Ordinary Labor and for other Purposes, dated 26 Sept 45. In: German Denazification Law and Implementations with American 
Militärbehörden in direkter Reaktion auf die Entnazifizierungskrise in Bayern (Entlassung Pattons und Schäffers) hastig konzipiert und sogleich in Kraft gesetzt, markiert zugleich Höhepunkt und Wende einer übermäßig expandierten Säuberungspraxis im amerikanischen Besatzungsbereich. Die Vorschriften zur Wirtschaftssäuberung übertrafen dort an Schärfe nun sogar die Richtlinien der Juli-Direktive zur Entnazifizierung der Verwaltung.

Der Charakter der Entnazifizierung der Wirtschaft in Südwürttemberg hing entscheidend von der materiellen Ausgestaltung der dehnbaren französischen Anweisungen durch das Staatssekretariat ab. Dort wurden die Anweisungen der Militärregierung das Beispiel Nordwürttembergs vor Augen - mit Erleichterung aufgenommen. Landesdirektor Kilpper ermahnte deshalb die Landräte, das Vertrauen der französischen Behörden nicht durch die Überziehung der festgesetzten Termine zu enttäuschen. Er suggerierte in der Diskussion über die Konsequenzen der vermögensrechtlichen Maßnahmen der Säuberungsbestimmungen schon die politische Linie in der Entnazifizierung der Wirtschaft, noch ehe der erste Untersuchungssausschuß seine Arbeit aufgenommen hatte. Er bemerkte, daß „Strafmaßnahmen wie Vermögensbeschlagnahmen nur selten vorkommen würden "147. Während die organisatorischen Vorbereitungen zum Aufbau der erstinstanzlichen Säuberungsgremien liefen ${ }^{148}$, ging man im Staatssekretariat daran, die französischen Richtlinien näher zu interpretieren und in praktikable Ausführungsbestimmungen für die KRUAS umzusetzen. Dabei kam es gegenüber den französischen Richtlinien zu einer wesentlichen Akzentverschiebung. Der Erlaß der Militärregierung sah den politisch motivierten Ausschluß aller Elemente vor, die in den zurückliegenden Jahren, nach allgemeiner Kenntnis, durch ihre Verbundenheit mit dem NS-System zu dessen Stabilisierung beigetragen hatten. Ihnen konnten der direkte Einfluß oder die Mittel ihrer Einflußnahme im politisch-gesellschaftlichen Bereich entzogen werden, die nach der plausiblen Formel in der MilitärregierungsDirektive „die Wiederherstellung der demokratischen Freiheiten und Einrichtungen in Deutschland“ gefährden konnten. Dieser Hauptgesichtspunkt fiel in den „Hinweisen und Erläuterungen" der Wirtschaftsdirektion an die KRUAS weg ${ }^{149}$; politische Belastung wurde vornehmlich auf quasi-kriminelle Tatbestände reduziert, die in dem bevorstehenden Verfahren gesühnt werden sollten. Die politische Säuberung, hieß es in dem Runderlaß, solle nicht ,an die Stelle früheren Unrechts neues Unrecht“ setzen. Vielmehr solle geschehenes Unrecht wieder gutgemacht werden, ,indem die Schuldigen zur Rechenschaft und Sühne herangezogen werden." Gleichgültig, ob jemand einer NS-Organisation angehört habe oder nicht, sollten es die KRUAS als ,entscheidend" ansehen, ob sich jemand, unter Ausnutzung politischer Beziehungen oder

Direktives included, $1^{\text {st }}$ edition, S. 204. Zu Vorgeschichte, Inhalt und Konsequenzen dieses Gesetzes, vergl. Griffith, Denazification Program, S. 91 ff. u. Niethammer, Entnazifizierung, S. 299 ff.; dort auch die Analyse der neu geschaffenen Revisionsmöglichkeit durch die „review boards“.

147 3. Lrtg in Reutlingen am 3. 1. 1946, S. 23.

148 Erlaß der LdW an alle Landräte v. 10. 12.1945; SIG, Wü 40/76/6000/3.

${ }^{149}$ Alle Zitate aus dem Runderlaß der LdW an alle Landräte v. 10.1.1946; SIG, Wü 15/514. Gebilligt in der DirS 8.1.1946. 
durch unmittelbaren politischen Druck, gegenüber Konkurrenten „unrechtmäßige Vorteile persönlicher oder finanzieller Art" verschafft hatte. Dabei galt die Denunziation als besonders schwerwiegendes Delikt. Die Einziehung von Vermögen als politische Maßnahme wurde den KRUAS praktisch ausgeredet. Sie sollten stattdessen Geldbußen ,als teilweise Vermögenskonfiskationen“ festsetzen; doch schon zur Verhängung dieser Maßnahme mußten die KRUAS dem Betroffenen die Umsetzung seiner politischen Beziehungen zu maßgeblichen Parteistellen in ,,ungerechtfertigte Berufs- oder Vermögensvorteile" nachweisen. Durch die Einengung des politischen Säuberungsverfahrens auf die Sühnung - in der Regel kaum nachweisbarer - persönlicher Schuld, verschüttete die Anweisung der Wirtschaftsdirektion die von strafähnlichen Erwägungen freien Intentionen der politischen Säuberung. So blieb den durch ihr politisches Engagement Kompromittierten, sofern sie sich keine „verwerflichen Handlungen" hatten zuschulden kommen lassen, eine gute Chance, auch nach dem Zusammenbruch des nationalsozialistischen Systems ihren gesellschaftlichen Einfluß zu behaupten. In den Fällen, die nach den Vorschriften in eine der Formalbelastungskategorien fielen, waren die KRUAS gehalten, jeden „Schematismus“ zu vermeiden und stattdessen die ,gesamte Haltung der Person“ zu würdigen.

Die Uberprüfung der freien Wirtschaft auf Kreisebene begann in etwa termingerecht Ende Januar $1946^{150}$. Die meisten Ausschüsse sonderten allerdings zunächst die Akten der Unbelasteten aus, so daß nach vierwöchiger Tätigkeit der Säuberungsorgane gewichtigere Fälle noch kaum vorlagen ${ }^{151}$. Ebenso wie bei der Beamtensäuberung, der die Militärregierung übrigens wiederholt die eindeutige Priorität gegenüber der Entnazifizierung der Wirtschaft einräumte ${ }^{152}$, war auch hier wieder die eine oder andere Berufsgruppe durch das Raster der Vorschriften gefallen und zunächst unbehelligt geblieben ${ }^{153}$. Die Einstufung der Säuberungsfälle durch die KRUAS konnte nicht innerhalb der Fristen abgeschlossen werden, obwohl auch hier die Anwesenheit der französischen Enquête-Kommission eine gewisse Forcierung brachte ${ }^{154}$. Erst Anfang April, als schon die grundlegende Neuordnung des gesamten Säuberungsverfahrens in Südwürttemberg eingeleitet war, konnte die Landesdirektion der Wirtschaft dem Gouvernement Militaire melden, daß „die Untersuchungsausschüsse der 1. Instanz ihre Arbeit zum größten Teil abgeschlossen haben, so daß demnächst die Säuberungskommissionen ihre Entscheidung ... treffen können" 155 .

Die statistischen Angaben über die Wirtschaftssäuberung in erster Instanz sind sehr

150 „Kurzer Wirtschaftsbericht über die Zeit vom 15.1. bis 15.2. 1946“ der LdW v. 19. 2. 1946; SIG, Wü 140/1019/Bd.I/4. DirS 22. 1. 1946.

151 Schreiben der LdW an alle Landräte v. 25. 2. 1946; SIG, Wü 15/514.

152 Mitteilung G. H. Müllers an Landesdirektor Roßmann über einen Telefonanruf des Säuberungskommissars der Milreg v. 21. 2. 1946; SIG, Wü 13/154.

153 Schreiben der LdW an das Landratsamt Biberach v. 1. 3. 1946; SIG, Wü 140/76/6000/26.

154 Schreiben der LdW an die Landratsämter v. 7. 2. 1946; SIG, Wü 140/76/6000/24.

155 „Bericht der Landesdirektion der Wirtschaft über die Lage der industriellen und gewerblichen Wirtschaft in der französisch besetzten Zone von Württemberg und Hohenzollern" an die Milreg v. 4. 4. 1946; SIG, Wü 140/1019/Bd. I/4. 
aussagearm. Insgesamt waren 7500 Fälle überprüft worden. In rund 73 Prozent aller Fälle lag nach Ansicht der KRUAS keine politische Belastung vor, 22 Prozent gehörten zur Gruppe $b$ und rund 5 Prozent wurden als c-Fälle eingestuft ${ }^{156}$.

Wenigstens die Formalbelastungslisten boten den KRUAS einige wenige Anhaltspunkte bei ihrer Entscheidungsfindung, ansonsten wurde allgemein das Fehlen ,konkreterer Vorschriften" beklagt ${ }^{157}$. Aus dem Tenor der Abschlußberichte ist herauszulesen, daß in der ersten Säuberungsinstanz kaum Vorschläge des Vermögenseinzugs bei besonders Belasteten gemacht worden sein dürften. Zum einen boten hierfür die Ausführungsbestimmungen vom 10. Januar 1946 kaum eine Handhabe, zum anderen reichte für solche Maßnahmen die politische Rückendeckung durch die Landesdirektion der Wirtschaft einfach nicht aus. So stellte ein führender Beamter der Wirtschaftsdirektion vor den KRUAS in Besprechungen zu Beginn der Untersuchungen noch einmal klar, daß mit der politischen Säuberung „lediglich der Sand aus den Rädern der Wirtschaft entfernt werden" solle $^{158}$. Es ist ein Indiz für die innere Unsicherheit, mit der sich mancher KRUA zu wirklich fühlbaren Sanktionen entschlossen haben mag, wenn es die Mitglieder eines Ausschusses für geboten hielten, darauf hinzuweisen, „daß wir uns mit unserem Urteil nicht privatrechtlich verantwortlich betrachten "159. Ähnlich wie bei der Verwaltungssäuberung zeigten die Ergebnisse, die die KRUAS vorlegten, selbst bei Berücksichtigung der unterschiedlichen wirtschaftlichen Struktur der Landkreise, wiederum erhebliche Schwankungen. Das ist aus der Behandlung der leichten Fälle ersichtlich, wo die Quote der in diese Kategorie eingestuften Fälle zwischen 26 Prozent ${ }^{160}$ und 94 Prozent $^{161}$ schwankte. Auch hier hätten die Säuberungskommissionen die Vorarbeiten der KRUAS in der zweiten Instanz desavouieren müssen ${ }^{162}$. Doch dazu ist es gar nicht mehr gekommen. Die Säuberungskommissionen konnten ihre Arbeit nicht mehr nach dem alten Erlaß beginnen; vor der Neuordnung der politischen Säuberung in Südwürttemberg fällten diese Gremien keine einzige Entscheidung mehr.

Die Aufstellung der drei Säuberungskommissionen für die freie Wirtschaft hatte Ende Dezember 1945 begonnen $^{163}$. Sie hatten ihren Sitz bei den Industrie- und Handelskammern Rottweil, Ravensburg und Reutlingen und waren für die jeweils umliegenden Kreise zuständig. Die Personalvorschläge zur Besetzung der Kommissionen gingen, nachdem man sie zuvor intern mit dem Chef der Wirtschaftsabteilung der Mili-

${ }^{156}$ Berechnet nach den KRUA-Wochenberichten an die LdW (Stand 26. 4. 1946); SIG, Wü 14/77/ 6000.

157 Tätigkeitsbericht des KRUA Tettnang an das Landratsamt v. 12. 4. 1946; SIG, Wü 140/76/6000. S.a. den Bericht des KRUA Wangen v. 15.3. 1946; SIG, Wü 140/77/6000.

158 Tätigkeitsbericht des KRUA Tettnang; ebd.

159 Tätigkeitsbericht des KRUA Wangen; ebd.

160 Landkreis Rottweil.

161 Landkreis Balingen.

162 Die Mitglieder des Tettnanger KRUA baten die LdW ihr die endgültigen Sanktionen mitzuteilen, da man sich ,mit jedem einzelnen Falle auseinandergesetzt" habe; ebd.

163 Schreiben der LdW an die Industrie- und Handelskammern v. 21.12.1945; SIG, Wü 140/76/ $6000 / 5$. 
tärregierung abgestimmt hatte ${ }^{164}$, Anfang April dem Gouverneur zur Genehmigung $\mathrm{zu}^{165}$. Gegen die Personalstruktur der Kommissionen wurden keine Einwände erhoben, auch von den Vertretern der drei Parteien nicht. Doch spätestens am Tage ihrer offiziellen Bestätigung durch die Militärregierung war den Kommissionen ihre Arbeitsgrundlage auch schon wieder entzogen. In einem Schreiben von Anfang April ${ }^{166}$ sprach sich jetzt auch der Gouverneur für den Vorschlag aus, der von Carlo Schmid schon vierzehn Tage zuvor im Direktorium angeregt worden war: eine grundlegende inhaltliche und organisatorische Neuordnung der Entnazifizierung in Südwürttemberg. Dadurch waren die bisherigen Bestimmungen obsolet geworden; die Entnazifizierung stagnierte für einige Wochen. So bestand im Frühjahr 1946 im französisch besetzten Gebiet Südwürttembergs bei der Säuberung der Wirtschaft eine erhebliche Diskrepanz zwischen Anspruch und Wirklichkeit. Einerseits waren seit der Besetzung des Landes im Bereich des Wirtschaftslebens durch offizielle Säuberungsgremien noch keinerlei Sanktionen ausgesprochen oder gar vollzogen worden, andererseits lag der Öffentlichkeit aber die denkbar radikale Erklärung vor, die der Gouverneur den Parteien abgepreßt hatte und in der von Entlassung und Enteignung von 50-75 Prozent politisch belasteter „Betriebsführer“ die Rede war.

Doch ebenso, wie diese drastische Resolution ohne praktische Konsequenzen blieb, kamen in diesen Wochen des zeitweiligen Stillstandes der Entnazifizierung auch solche Vorstellungen nicht zum Tragen, die die ohnehin höchst zurückhaltende Wirtschaftssäuberung vollends hätten zur Farce werden lassen ${ }^{167}$. Ebenso blieben unter den gegebenen Umständen die Richtlinien für die Arbeit der Säuberungskommissionen, die die Landesdirektion der Wirtschaft schon ausgearbeitet hatte, in der Schublade. Aus ihnen erhellt sich aber noch einmal exemplarisch die Konzeption, mit der die Wirtschaftsdirektion dem Säuberungsverfahren die Spitze abzubrechen versuchte. Noch deutlicher als die Vorschriften für die KRUAS lassen die Richtlinien für die Entscheidungsfindung in den Kommissionen erkennen, wie die Entnazifizierung der Wirtschaft in ihrer Stoßrichtung umgelenkt und damit ihres politischen Kerns entklei-

164 Unterredung des Chefs der Wirtschaftsabteilung der Militärregierung mit den Kommissionsmitgliedern in Ravensburg. Schreiben des Landrates des Kreis Ravensburg an LdW v. 20. 3. 1946; SIG, Wü 140/76/6000.

165 Schreiben des Landesdirektor Dr. Kilpper an die Milreg v. 3. 4. 1946; SIG, Wü 140/76/6000. Die „Zahl der zur Verfügung stehenden, nach Persönlichkeit und politischer Belastung geeigneten Personen“ war gering, zumal ein „nicht unwesentlicher Teil dieser Herren eine Beteiligung an der Kommission grundsätzlich ablehnt[e]." Schreiben der IHK Ravensburg an LdW v. 29.1. 1946; ebd.

166 Schreiben von Gouverneur Widmer an das Staatssekretariat v. 6. 4. 1946; SIG, Wü 80, acc. 10/ $1974,56$.

167 In einer Direktoriumssitzung (8.1.1946) schlug Landesdirektor Dr. Paul Binder (später CDU) vor, den Eigentümern, die durch das Säuberungsverfahren zur Aufgabe ihres Vermögens gezwungen würden, die Möglichkeit offen zu lassen, „,den Besitz an Verwandte zu verkaufen“. In einer Auskunft an einen Säuberungsausschuß vertrat die LdF in einer ähnlichen Frage die Auffassung, ein Betriebsleiter, dem die "Eignung zur Leitung eines Betriebes dauernd abgesprochen" sei, könne die Geschäftsleitung jederzeit an seine Ehefrau (!) übergeben. Schreiben der LdF an den Landrat von Reutlingen v. 15. 1. 1946; SIG, Wü 140/76/6000. 
det werden sollte. Als Zielgruppe rückten nun unter der Hand die quasi-kriminellen Elemente des Wirtschaftslebens in den Mittelpunkt des Verfahrens, die „durch Ausnützung politischen Einflusses oder politischer Beziehungen ungerechtfertigte Vorteile erlangt, andere Menschen unrechtmäßig geschädigt oder sich übler Denunziationen schuldig gemacht" hatten ${ }^{168}$. Diese Akzentuierung, die ganz auf der Linie des bereits erwähnten Erlasses an die Kreisausschüsse lag, mußte eine starke Verengung des Betroffenenkreises nach sich ziehen. Die Möglichkeit zur Relativierung jeglicher Belastung und Entpolitisierung der politischen Säuberung gab dann die Passage des Entwurfes, in dem es hieß: „Es kommt vielmehr entscheidend immer auf das gesamte Verhalten der zu überprüfenden Person an."

Immerhin wurde dieses in der Wirtschaftsdirektion offenbar vorherrschende Entnazifizierungsverständnis nicht von allen Beamten der Behörde geteilt. In seinen „Bemerkungen zu dem Entwurf von Richtlinien für die Säuberungskommission der Wirtschaft" setzte sich ein Kritiker mit dieser restriktiven Auffassung auseinander. In dem ungezeichneten Kommentar wurde der eigentliche Sinn der Säuberungsaktion herausgestellt. Dort hieß es, die Richtlinien für die Kommission müßten als Ziel der politischen Säuberung „die Notwendigkeit herausstellen, von den leitenden Posten der Wirtschaft die Personen zu entfernen, deren Haltung gezeigt hat, daß sie Überzeugungen hegen, die den demokratischen Grundsätzen so sehr widersprechen, daß man ihnen in dem neuen Deutschland kein Vertrauen mehr entgegen bringen kann." Ihnen müßten deshalb die „Beeinflussungsmittel“ entzogen werden, die das „Eigentum an einem Unternehmen" nun einmal böten. Klar war gegenüber dem Richtlinien-Entwurf auch herausgestellt: „Die Säuberungsaktion hat daher nicht das Ziel, Verfehlungen (politische Verfehlungen oder Verstöße gegen das Völkerrecht) zu bestrafen"169. Deshalb war in dem Kommentar vorgeschlagen, Satz für Satz alle Passagen aus dem Entwurf zu entfernen, die darauf abzielten, die Entnazifizierung der Wirtschaft zu einer ihres politischen Sinnes entkleideten Strafaktion zu degradieren.

168 Richtlinien-Entwurf der LdW für die Säuberungskommissionen der Wirtschaft; SIG, Wü 140/76/ 6000. Ebd. 